Ger J Exerc Sport Res 2020 · 50:280-296 https://doi.org/10.1007/s12662-020-00654-x Eingegangen: 26. September 2019

Angenommen: 6. April 2020

Online publiziert: 29. April 2020

(c) Der/die Autor(en) 2020

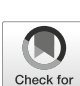

Lutz Thieme

Hochschule Koblenz, Remagen, Deutschland

\title{
Jung stirbt, wen die Götter lieben?
}

\section{Zur Mortalität deutscher Olympiateilnehmer 1956 bis 2016}

Die nachhaltig positiven gesundheitlichen Wirkungen von Bewegung mit moderater Intensität beispielsweise bei breitensportlichen Aktivitäten sind mittlerweile gut belegt (Blair et al., 1995; Kujala, Kaprio, Sarna, \& Koskenvuo, 1998; Turi-Lynch et al., 2019) und werden aktuell weder von Experten noch im öffentlichen Diskurs ernsthaft bestritten. Dagegen sind die Erkenntnisse und die Datenlage zu den langfristigen gesundheitlichen Folgen von sportlichen Belastungen, wie sie typischer Weise im Spitzensport auftreten und sich letztlich in der Lebenserwartung der Gruppe der Leistungssportlerinnen und Leistungssportler niederschlagen, deutlich schmaler und heterogener. Durch die mediale Berichterstattung über Todesfälle ehemaliger Spitzenathletinnen und -athleten kann der Eindruck entstehen, dass diese einem höheren Sterblichkeitsrisiko ausgesetzt sind, als dies für die vergleichbare Gesamtbevölkerung der Fall ist. In Deutschland gewinnen Erkenntnisse zur Mortalität von Spitzensportlern vor dem Hintergrund der Debatte um die gesundheitlichen Folgen von Dopingpraktiken in beiden deutschen Staaten, den Dopingopfer-Hilfegesetzen zur Gewährung von Hilfeleistungen für DDR-Dopingopfer (Bundestag, 2002a, b, 2016), den Diskussionen zu deren Missbrauch (Knuth, 2018; Sturmberg, 2018) sowie angesichts der Planungen einer sogenannten „Sportlerrente“ (Sturmberg \& Freitag, 2019) zusätzlich an Bedeutung. Daher ist es erstaunlich, dass bislang nur einzelne Untersuchungen zur Mortalität von Spitzensportlern in Deutschland vorliegen, obwohl derartige Analysen international durchaus $\mathrm{zu}$ finden sind, teilweise überraschende Befunde berichtet werden (z. B. Becker, Chay, \& Swaminathan, 2007) und Mortalitätsdaten als schnellster und zuverlässigster Indikator für die Lebensdauer („longevity“; Vaupel, 2010, 536) gelten. Gerade die Unvollständigkeit und die Heterogenität zwischen den jeweiligen Erhebungszeiträumen, den Untersuchungsgruppen, den mit der Herkunftsnation verbundenen sozioökonomischen Einflüssen sowie den Anforderungen der Sportarten erweisen sich als Hindernisse bei der Entwicklung von Theorieelementen, die Einflüsse von Spitzensport in ihrer Wechselwirkung $\mathrm{zu}$ anderen mortalitätsbeeinflussenden Faktoren wie beispielsweise Bildung und sozialer Status erklären. Die folgende Untersuchung möchte die vorhandene Datengrundlage erweitern und helfen, Erkenntnislücken zu reduzieren, indem zunächst der Forschungsstand zur Mortalität von Spitzensportlern rezipiert wird. Aus den bisherigen Befunden werden Thesen ${ }^{1}$ abgeleitet, die die bisherige Datenlage zusammenfassen. Dem folgt die Darstellung der zur Organisation der erhobenen Daten verwendeten statistischen Verfahren. Der Vergleich zwischen der Beschreibung des Phänomens „Mortalität von Spitzensportlerinnen und -sportlern“ mit den neu gewonnenen Daten einerseits sowie der bislang vor-

\footnotetext{
1 Da Hypothesen in der Regel bereits den Zusammenhang zwischen Variablen beinhalten, wird der weniger spezifische Begriff der These verwendet.
}

liegenden Datengrundlage andererseits schließt sich an. Die gewonnenen explorativen Ergebnisse werden diskutiert bevor der Beitrag mit einer Zusammenfassung, die auch die Limitationen benennt und einen Ausblick auf weiteren Forschungsbedarf gibt, schließt.

\section{Forschungsstand}

Zur Abschätzung langfristiger gesundheitlicher Wirkungen von Spitzensport werden Überlebenszeitanalysen genutzt. Hierbei werden die Überlebenschancen von Spitzensportlerinnen und Spitzensportlern eines Landes sowie die jeweiligen Todesursachen mit denen der vergleichbaren Gesamtpopulation verglichen oder die Überlebenschancen innerhalb verschiedener Teilpopulationen von Spitzensportlerinnen und Spitzensportlern untersucht. Gängige Vergleichsmaße sind dabei die standardisierte Mortalitätsrate („standardised mortality ratio" [SMR]; Bland, 2015, 349f.) sowie Überlebenskurven bzw. -funktionen (Altman, 1999, 368 ff.). Die SMR gibt das Verhältnis zwischen den Sterberisiken einer $\mathrm{zu}$ beurteilenden Gruppe mit einer Referenzgruppe als Faktor oder als Prozentsatz an. Überlebenskurven sind die abwärts kumulierten Häufigkeiten der Überlebenden einer Population im Zeitverlauf (Gaus \& Muche, 2014, 190 ff.).

Um Erkenntnisse zu gesundheitlichen Wirkungen spitzensportlichen Engagements zu erhalten, nutzen alle nachfolgend rezipierten Studien die Daten von Teilnehmerinnen und/oder Teilnehmern 
an spezifischen Spitzensportereignissen (Olympische Spiele, Tour de France), an Ligawettbewerben (National Football League, National Baseball League) oder sogar nur von Medaillengewinnerinnen und -gewinnern bzw. Finalistinnen und Finalisten spitzensportlicher Großereignisse, da damit das Abgrenzungsproblem zwischen Breiten- und Spitzensport gelöst wird. Teramoto und Bungum (2010) identifizieren in einem entsprechenden Review insgesamt 14 Studien, die Teilnehmerinnen und Teilnehmer an nationalen oder internationalen Meisterschaften bzw. professioneller Ligen mit der Gesamtbevölkerung hinsichtlich Sterberisiko oder Überlebenschance vergleichen. Die Ergebnisse zeigen konsistente Ergebnisse für Sportarten, die auf vorrangig aerober Energiebereitstellung basieren (Ausdauersportarten) sowie für Sportarten mit einer Mischung aus aerober und anaerober Energiebereitstellung („mixed sports“). Die jeweils beobachtete Spitzensportpopulation hat eine höhere Lebenserwartung und eine geringere standardisierte Mortalitätsrate im Vergleich zur jeweiligen nationalen Gesamtbevölkerung. Die Autoren führen diesen Effekt auf die positiven kardiovaskulären Wirkungen der Sportausübung zurück.

Für Sportarten, die vor allem auf anaerober Energiebereitstellung basieren (Kraftsportarten) sowie für Mannschaftssportarten finden sich differenzierte Ergebnisse. Professionelle italienische Fußballer der ersten drei Ligen der Jahre 1960 bis 1996 verfügen über keine Überlebensvorteile gegenüber der Gesamtbevölkerung (Belli \& Vanacore, 2005). Ähnliche Ergebnisse finden sich für 822 Mitglieder der neuseeländischen Rugby-Nationalmannschaft („All Blacks") seit 1884 (Beaglehole \& Stewart, 1983). Mit den Ergebnissen von Abel und Kruger $(2005,2006)$ lässt sich postulieren, dass Überlebensvorteile von Spitzensportlerinnen und -sportlern in den Mannschaftssportarten in weiter zurückliegenden Perioden nicht nachzuweisen sind, während sich die Befunde mehren, die eine geringere SMR in Peri- oden nach den 1950er Jahren feststellen². Im Gegensatz zu den Ausdauersportlern und den "mixed sports“ findet sich für die finnischen Meister im Gewichtheben der Jahre 1977-1982 eine höhere Mortalität in den nachfolgenden 12 Jahren, verglichen mit der Gesamtbevölkerung. Als Ursache vermuten die Autoren die Verwendung von Anabolika (Pärssinen, Kujala, Vartiainen, Sarna, \& Seppäla, 2000).

Die von Teramoto und Bungum (2010) gesammelten Befunde wurden in den vergangenen Jahren durch weitere Studien hinsichtlich (I) der Mortalität von Spitzensportlerinnen und -sportlern im Vergleich zur Gesamtbevölkerung, (II) der Differenz zwischen Sportarten mit aerob bzw. anaerob geprägter Energiebereitstellung, (III) der Mortalität in Mannschaftssportarten, (IV) der vermuteten Wirkung von Doping, (V) des Vergleichs von Gewinnern und Platzierten sowie (VI) der Unterschiede zwischen Athletinnen und Athleten angereichert. Alle Studien berichten zwar Effekte auf die Mortalität der jeweiligen Untersuchungsgruppe, nur in wenigen Fällen werden jedoch Erklärungen für die beobachteten Phänomene ad hoc vorgeschlagen. Diese Erklärungsansätze erschöpfen sich zudem im Verweis auf positive kardiovaskuläre Wirkungen, höhere Raten von Herzerkrankungen und Suiziden sowie vermutete Dopingfolgen. Die ermittelten Befunde werden explorativ miteinander verknüpft, eine theorie- oder modellprüfende Methodologie findet sich in keinem der Beiträge. Dies dürfte mit den methodologischen und methodischen Schwierigkeiten zusammenhängen, die Wirkung isolierter Faktoren auf die Lebensspanne auf individueller und kollektiver Ebene trennscharf zu bestimmen. Letztlich ginge es um eine Theorie, die sämtliche Lebensereignisse in Bezug auf ihr jeweiliges Risiko für die Lebensspanne ursächlich erklärt. Dies geht weit über physiologische Erklärungsansätze zum höheren Risiko einer Gruppe, an einem bestimmten Krankheitsbild zu sterben, hinaus

\footnotetext{
2 Bei Abel und Kruger $(2005,2006)$ tritt dieser Effekt bereits in den Betrachtungszeiträumen 1901 bis 1930 und 1931 bis 1973 auf.
}

(vgl. dazu jüngst Mackay et al. (2019) zum Risiko schottischer Profifußballer, an neurodegenerativen Krankheiten zu versterben).

Daher wird im vorliegenden Beitrag versucht, durch einen Vergleich der bislang vorliegenden Ergebnisse zur Mortalität von Spitzensportlerinnen und -sportlern mit Ergebnissen zur Mortalität deutscher Olympiateilnehmerinnen und -teilnehmer der Jahre 1956 bis 2016 sowie mit der Gesamtbevölkerung in beiden Teilen Deutschlands zu ergründen, ob sich die deutschen Daten in den bisherigen Forschungsstand einfügen und zu diskutieren, worin die Ursachen abweichender Datenlagen begründet sein könnten.

\section{Mortalität von Spitzensportler- innen und -sportlern vs. Gesamtbevölkerung}

Über die von Teramoto und Bungum (2010) angegebenen Befunde hinaus liegen mittlerweile weitere Studien vor, die eine geringere Mortalität von Olympiateilnehmern im Vergleich zur Gesamtbevölkerung ausweisen. So stellen Clarke et al. (2012) fest, dass die olympischen Medaillengewinner der Jahre 1896 bis 2010 aus neun Ländern durchschnittlich 2,8 Jahre länger leben als die vergleichbare Gesamtbevölkerung. Die 233 männlichen kroatischen Olympiamedaillengewinner der Jahre 1948 bis 1988 (für Jugoslawien) und von 1992 bis 2016 (für Kroatien) weisen ebenfalls eine signifikant niedrigere Mortalität $(\mathrm{SMR}=0,73$; $p<0,05)$ auf als die alters- und zeitkontrollierte Gesamtbevölkerung (Radonić et al., 2017). Für 1273 männliche polnische Olympiateilnehmer der Jahre 1924 bis 2010, die den zweiten Weltkrieg überlebt haben, finden Lin, Gajewski, und Poznańska (2016) sowohl für die Kohorte der vor 1920 Geborenen als auch für die danach Geborenen ein verringertes Sterberisiko im Vergleich zur Gesamtbevölkerung (Gajewski \& Poznańska, 2008). Für französische Olympioniken der Spiele von 1948 bis 2010 berichten Antero-Jacquemin et al. (2015) von einer standardisierten Mortalitätsrate von 0,49 für die Athletinnen ( $n=601$; 13 Todesfälle bis Studienende) und 0,51 
für die Athleten ( $n=1802 ; 222$ Todesfälle bis Studienende) und damit von signifikanten Unterschieden zur Gesamtbevölkerung. Auch 203 französische Teilnehmer an den olympischen Ruderwettbewerben der Jahre 1912 bis 2012 weisen sowohl in der Kohorte 1912 bis 1936 $(\mathrm{SMR}=0,63)$ als auch in der Kohorte 1948 bis $2012(\mathrm{SMR}=0,40)$ eine im Vergleich zu Gesamtbevölkerung signifikant geringere Mortalität auf (Antero-Jacquemin et al., 2014). Vergleichbare Differenzen zur Gesamtbevölkerung bestehen auch bei 786 französischen Teilnehmern an der Tour de France 1947 bis 2012 $(\mathrm{SMR}=0,59$; Marijon et al., 2013). Eine Metaanalyse von 10 Studien (Garatachea et al., 2014) und Reviews von Kujawska et al. (2017) sowie Lemez und Baker (2015), die alle teilweise bereits bei Teramoto und Bungum (2010) referierte Studien berücksichtigen, kommen zu vergleichbaren Ergebnissen.

Die Daten für die Bundesrepublik Deutschland wären mit dem internationalen Forschungsstand vereinbar, wenn gilt:

(T1) Deutsche Olympiateilnehmerinnen und -teilnehmer weisen eine niedrigere Mortalität im Vergleich zur Gesamtbevölkerung auf.

\section{Sportarten mit aerob bzw. anaerob geprägter Energiebereitstellung}

In Übereinstimmung mit den bisherigen Befunden stellen Lee-Heidenreich, LeeHeidenreich, und Myers (2017) Differenzen im Mortalitätsrisiko der olympischen Finalistinnen und Finalisten der Olympischen Spiele von 1928 bis 1948 im Hochsprung, im Diskuswerfen, im Marathon und im 100-m-Lauf fest. Diskuswerfer weisen das höchste Mortalitätsrisiko auf (zusammenfassend für Ausdauersportler vgl. Ruiz, Fiuza-Luces, Garatachea, \& Lucia, 2013), was konform zu den Befunden über positive kardiovaskuläre Wirkungen ausdauerorientierter Bewegung (Blair et al., 1995; Last \& Weisser, 2015) ist. Derartige Wirkungen sollten auch in Deutschland zu beobachten sein, sodass gelten sollte:

Ger J Exerc Sport Res 2020 · 50:280-296 https://doi.org/10.1007/s12662-020-00654-x

(c) Der/die Autor(en) 2020

\section{Thieme}

\section{Jung stirbt, wen die Götter lieben? Zur Mortalität deutscher Olympiateilnehmer 1956 bis 2016}

\section{Zusammenfassung}

Während moderate sportliche Bewegung die Überlebenswahrscheinlichkeit einer Risikogruppe positiv beeinflusst, sind die Befunde im Leistungssport international gemischt. Für Deutschland liegt dazu nur eine Studie zu Fußball-Nationalspielern vor. Daher wurden mit Hilfe der Daten der Mitglieder der deutschen Olympiamannschaften 1956 bis 2016 ( $n=6066)$ Hypothesen zu Effekten im Vergleich zur Gesamtbevölkerung, zum metabolischen Stoffwechsel, zu Einzel-, Mixund Mannschaftssportarten, zu Doping, zum olympischen Erfolg und zum Geschlecht getestet und dabei auch die Mitgliedschaft in den Olympiamannschaften der Bundesrepublik, der DDR sowie dem wiedervereinigten
Deutschland berücksichtigt. Es zeigt sich, dass die Mitglieder der deutschen Olympiamannschaften bislang eine im Vergleich mit der Gesamtbevölkerung höhere Mortalitätsrate aufweisen, es keine höheren Überlebensraten für die Mitglieder der Olympiamannschaften der alten Bundesrepublik gibt, sich keine Differenzen zwischen Sportarten finden lassen, aber steigende olympische Erfolge zu höheren Mortalitätsraten führen.

\section{Schlüsselwörter}

Epidemiologie · Lebensdauer · Spitzensportler · Olympische Spiele · CoxRegression

\section{Whom the gods love die young? The mortality of German Olympic participants from 1956 to 2016}

\section{Abstract}

While moderate exercise has a positive influence on the life expectancy of a risk group, there are mixed findings on the impact of competitive sports on health. For Germany, there has only been one study on football (soccer) players of the national team. Thus, based on the data of the members of the German Olympic teams from 1956 to 2016 $(n=6066)$, different hypotheses were tested, such as the longevity in comparison to the total population, the effects on athletes' metabolism, the differences between individual, mixed and team sports, and the effects of doping, Olympic success and gender. Membership in the Olympic teams of the Federal Republic of Germany
(FRG), the German Democratic Republic (GDR) and reunited Germany was also taken into account. The analysis shows that members of the German Olympic teams have a higher mortality rate compared to the total population. Members of the Olympic teams of the former FRG do not show higher survival rates than those from the GDR. Furthermore, no differences between types of sports can be found, but there appears to be a positive correlation between Olympic success and mortality rates.

Keywords

Epidemiology · Longevity · Elite athletes . Olympic Games · Cox regression
(T2) Deutsche Olympiateilnehmerinnen und -teilnehmer in von anaerobem Stoffwechsel geprägten Sportarten weisen ein im Vergleich zu aerob geprägten Sportarten höheres Mortalitätsrisiko auf.

\section{Mannschaftssportarten}

Die Befundlage zur Wirkung von Mannschaftssportarten ist uneinheitlich. Kuss, Kluttig, und Greiser (2011) vergleichen alle Deutschen Fußball-Nationalspieler der Jahre 1908 bis $2006(n=812)$ mit der
Gesamtbevölkerung. In allen betrachteten 13 Zeitintervallen waren die beobachteten Todesfälle der Nationalspieler höher als aus den Daten der Gesamtbevölkerung zu erwarten war. Je jünger ein Spieler bei der Erstberufung in die Nationalmannschaft war, desto höher war das Risiko, eher als erwartet zu versterben. Venkataramani, Gandhavadi, und Jena (2018) untersuchen 3812 Spieler der National Football League (NFL) der Spielzeiten 1982 bis 1992 und finden keine signifikanten Unterschiede zur Bevölke- 
rung (DeKosky, Jaffee, \& Bauer, 2018 zur Diskussion der Ergebnisse). Dagegen berichten Lehman et al. (2012) in Bezug auf eine Kohorte von 3439 NFL-Spielern der Spielzeiten 1959 bis 1988 von einer insgesamt niedrigeren Mortalität bei gleichzeitig deutlich höherem Risiko, an neurodegenerativen Erkrankungen zu versterben. Bei Spielern der Spielzeiten 1937 bis 1975 mit mehr als drei Spielzeiten ist das Mortalitätsrisiko $40 \%$ höher als bei Spielern bis zu drei NFLSpielzeiten (Williams, 2012). Um Selektionseffekte zu kontrollieren, vergleichen Nguyen et al. (2019) 3419 Spieler, die zwischen 1959 und 1988 ihre letzte NFLSaison spielten, mit 2708 Spielern der Major League Baseball (MLB), die der NFL-Kohorte angepasst wurden. Auch hier ist das Mortalitätsrisiko für die NFLKohorte signifikant höher. Dagegen ermitteln Lemez, Wattie, und Baker (2018) für MLB-Spieler das höchste Mortalitätsrisiko unter allen Spielern der amerikanischen Major Leagues ${ }^{3}(n=50.515)$. Einen umfassenden Vergleich zwischen afroamerikanischen, Hispanics und weißen Spielern der MLB in den Geburtenjahrgängen 1905 bis 1966 legt Markowitz (2019) vor.

Die aktuell uneinheitliche Befundlage schließt nicht aus, dass Mannschaftssportarten anderen Wirkungen auf das Mortalitätsrisiko ausüben als Einzelsportarten, zumal Hinweise auf Differenzen in den Persönlichkeitsmerkmalen zwischen Einzel- und Mannschaftssportlerinnen und Sportlern vorliegen (Eagleton, McKelvie, \& de Man, 2007). Dies führt zu folgender These:

(T3) Deutsche Olympiateilnehmerinnen und -teilnehmer in Mannschaftssportarten weisen eine höhere Mortalität im Vergleich zu den Teilnehmern in den Einzelsportarten auf.

\section{Doping}

Für die männlichen Olympiasieger im Ringen der Jahre 1896 bis 2016 stellt Keller (2019) fest, dass diese unabhän-

\footnotetext{
${ }^{3}$ Neben der MLB und der NFL sind dies die National Basketball Association (NBA) und die National Hockey League (NHL).
}

gig vom Herkunftsland, der Gewichtsklasse, weiterer Olympiateilnahmen oder Phasen, in denen die Einnahme anaboler Steroide vermutet wird, im Vergleich zur Gesamtbevölkerung im Durchschnitt gut 19 Jahre länger leben. Lindqvist et al. (2013) wählen eine Kohorte männlicher schwedischer Spitzensportler der Sportarten Kraftdreikampf (Powerlifting), Gewichtheben sowie der leichtathletischen Wurfdisziplinen, die Platz 1 bis 10 der schwedischen Rangliste der Jahre 1960 bis 1979 einnahmen $(n=1199)$, weil sie in diesem Zeitraum und in diesen Disziplinen den Gebrauch anaboler Steroide vermuten. Sie beobachten keine gestiegene Mortalität im gesamten Beobachtungszeitraum gegenüber der männlichen Gesamtbevölkerung, jedoch eine deutlich höhere Mortalität im Lebensalter von 20 bis 50 Jahren. Auch die Selbstmordrate der Athleten war im Alter von 30 bis 50 Jahren spürbar erhöht.

In Deutschland ist die Verwendung von Doping sowohl für die Bundesrepublik als auch für die DDR nachgewiesen. Unterschiede werden vor allem in der systematischen, staatlich geplanten und unterstützten Anwendung in der DDR gesehen, was zur Verabschiedung des ersten und zweiten Dopingopfer-Hilfegesetzes beitrug, das sich auf ehemalige DDR-Kaderathletinnen und -athleten beschränkt (Bundestag 2002a, b, 2016). In der Gesetzesbegründung für das erste Dopingopfer-Hilfegesetz heißt es dazu u. a., dass der Einsatz der Dopingmittel in der DDR ab 1966 vorwiegend bei weiblichen Sportlern erfolgte, die Nebenwirkungen seit Anfang der 70er Jahre, spätestens ab 1975 bekannt gewesen seien und die Aussetzung der Applikationen nach den Olympischen Spielen 1976 zwar angeordnet, aber praktisch folgenlos blieb. Betroffen wären vor allem die Sportarten Leichtathletik, Rudern, Gewichtheben, Skilanglauf, Biathlon und Eiskunstlauf. Explizit wird auf die Langzeitschäden verwiesen, die durch die Verabreichung von Anabolika und Wachstumshormonen entstehen (Bundestag, 2002b). Die Liste der Sportarten mit den meisten Aberkennungen von olympischen Medaillengewinnen infolge nachgewiesenen Dopings wird dann auch von der Leichtathletik, dem Gewichtheben, dem Ringen und dem Skilanglauf angeführt ${ }^{4}$.

Allerdings können sich derartige Aussagen nicht auf Mortalitätsstudien stützen, da außer den Studien von Keller (2019) im Ringen sowie der Berücksichtigung deutscher Medaillengewinner der Olympischen Spiele 1896 bis 2010 bei Clarke et al. (2012) bislang keine deutschen Befunde verfügbar sind und die beiden international verfügbaren Studien keine Wirkung auf das Mortalitätsrisiko zeigen. Es soll jedoch in Übereinstimmung mit der politischen Bewertung die These geprüft werden, dass

(T4) die Mortalität ostdeutscher Olympiateilnehmerinnen und -teilnehmer höher ist als die der westdeutschen Olympiateilnehmerinnen und -teilnehmer. sowie

(T5) die Mortalität deutscher Olympiateilnehmerinnen und -teilnehmer in den Sportarten Leichtathletik, Rudern, Gewichtheben, Skilanglauf, Biathlon, Ringen und Eiskunstlauf höher ist als die der Olympiateilnehmerinnen und -teilnehmer in den anderen Disziplinen.

\section{Sieger vs. Platzierte}

Bei einem Vergleich der olympischen Gold- und Silbermedaillengewinner der Jahre 1896 bis 1948 in der Leichtathletik stellt Leive (2018) fest, dass die Goldmedaillengewinner mehr als ein Jahr früher als die Silbermedaillengewinner verstorben sind. Der Autor führt dies darauf zurück, dass die Silbermedaillengewinner im Lebensverlauf einen höher bezahlten Beruf erreichten und so den verpassten Olympiasieg materiell und sozial kompensieren konnten. Dem entgegen stehen die Befunde von Becker et al. (2007), die im Zusammenhang mit der Aufnahme in die Baseball Hall of Fame feststellen, dass aufgenommene und deutlich gescheiterte Spieler $10 \%$ länger leben als knapp gescheiterte Spieler (vgl. bereits Medvec, Madey, \& Gilovich, 1995). Auch die Befunde von Kalwij (2018), der die Lebensspanne

\footnotetext{
${ }^{4}$ Vgl. https://de.m.wikipedia.org/wiki/Liste_ der_aberkannten_olympischen_Medaillen.
} 
US-amerikanischer Medaillengewinner der Olympischen Spiele von 1904 bis 1948 untersuchte und keine Differenz zwischen den Gold- und Bronzemedaillengewinnern, jedoch ein im Vergleich dazu zwischen 2,4 und 3,9 Jahren kürzeres Leben der Silbermedaillengewinner fand, weisen in Richtung eines höheren Mortalitätsrisikos bei verpassten Siegen.

Für Deutschland wäre daher zu prüfen, ob sich das von Kalwij (2018) beobachtete Bewältigungsmuster zeigt. Dann müsste gelten:

(T6) Deutsche Silbermedaillengewinnerinnen und -gewinner bei Olympischen Spielen haben eine höhere Mortalität als Gold- und Bronzemedaillengewinnerinnen und -gewinner.

\section{Athletinnen vs. Athleten}

Die Befunde von Coate und Sun (2012) für 2099 Olympische Medaillengewinner seit 1900 sowie für 302 Finalisten der britischen und US-amerikanischen Tennis-Meisterschaften seit den 1880er Jahren weisen eine Differenz der Mortalität zwischen Athletinnen und Athleten auf. Sowohl in einer Teilkohorte der vor 1920 Geborenen $(n=421)$ als auch in der Gesamtkohorte findet sich eine 5 bis 7 Jahre längere Lebenszeit für die olympischen Athletinnen. Dieser Befund wird durch die Tenniskohorte gestützt. Allerdings werden beide Ergebnisse nicht im Verhältnis zur längeren Lebenszeit von Frauen gegenüber Männern in der jeweiligen Gesamtbevölkerung (zu deren unterschiedlicher Körperwahrnehmung vgl. bereits Boltanski, 1976) betrachtet. Ein spezifischer Nachteil von Athletinnen oder Athleten im Spitzensport wäre ja nur dann gegeben, wenn sich das Verhältnis der Mortalitätsraten aus der Gesamtbevölkerung im Spitzensport nicht wiederfindet.

Für Deutschland sollte daher zunächst gelten:

(T7) Deutsche Olympiateilnehmerinnen haben im Vergleich zu deutschen Olympiateilnehmern eine geringere Mortalität.

Wenn keine geschlechtsspezifischen Faktoren im Spitzensport (z. B. höheres
Dopingrisiko für Frauen) in Deutschland gewirkt haben, müsste

(T8) das Verhältnis der Mortalitätsraten von deutschen Olympiateilnehmerinnen und -teilnehmern dem Verhältnis der Mortalitätsraten von Frauen und Männern in der Gesamtbevölkerung entsprechen.

\section{Methoden}

Die Daten zu den deutschen Olympiateilnehmerinnen und -teilnehmern sind der Website https://www.sportsreference.com/olympics/ entnommen. Die dortigen Angaben wurden mit Wikipedia (z.B. für die westdeutschen Teilnehmer an den Olympischen Winterspielen 1972 https://de.wikipedia.org/ wiki/Olympische_Winterspiele_1972/ Teilnehmer_(BR_Deutschland)) sowie einschlägigen Chroniken Olympischer Spiele bei nicht plausiblen Angaben sowie stichprobenartig bei ca. $25 \%$ der Datensätze gegengeprüft. Bei Abweichungen zwischen zwei Quellen erfolgten weitere Recherchen, beginnend bei den in Wikipedia angegebenen Links. Die Fehlerquote der geprüften Datensätze lag unter $3 \%$ und betraf vorrangig Abweichungen in den Geburts- und Sterbedaten von wenigen Tagen. Dennoch kann nicht ausgeschlossen werden, dass einzelne Sterbefälle nicht erfasst wurden. Die Fehlerrate sollte aber eher klein sein und $\mathrm{zu}$ keiner systematischen Verzerrung zwischen den einzelnen Teilkohorten führen.

Um die entwickelten Thesen zu testen, wurden die Daten aller Olympiateilnehmerinnen und -teilnehmer der Jahre 1956 bis 2016 genutzt. Von der Verwendung der Daten vor dem zweiten Weltkrieg wurde aufgrund der durch diesen sowie den ersten Weltkrieg hervorgerufenen Effekte abgesehen, da diese nicht zu kontrollieren sind. Zudem sind die Daten zum Tod der Olympiateilnehmerinnen und -teilnehmer in der ersten Hälfte des 20. Jahrhunderts lückenhafter und unzuverlässiger als danach. Das Jahr 1952 wurden nicht berücksichtigt, da zu den Spielen in Helsinki (Sommer) und Oslo (Winter) nur eine westdeutsche Mannschaft sowie eine Mannschaft der Saar antrat. Insgesamt wurden durch Addition der Olympiamannschaften für den Zeitraum 1956 bis 2016 insgesamt 8934 deutsche Olympiateilnehmerinnen und -teilnehmer identifiziert. Reduziert um an mehreren Olympischen Spielen Teilnehmende, ergeben sich 6066 Personen, von denen 4023 einmal an Olympischen Spielen, 1436 an zwei, 442 an drei und 165 an vier und mehr Olympischen Spielen teilnahmen. Die beobachtete Kohorte von 6066 Olympiateilnehmerinnen- und -teilnehmern lässt sich in die Athletinnen und Athleten differenzieren, die für die DDR bzw. für die BRD in deren selbstständiger Mannschaft oder in der gemeinsamen deutschen Mannschaft bis 1964 antraten, sowie diejenigen, die nach der deutschen Wiedervereinigung für Deutschland starteten. Die gewonnenen 6066 Datensätze enthalten die Variablen Name, Vorname, Geschlecht, Geburtsdatum, Sterbedatum, Jahr der Olympiateilnahme(n), Sportart, Medaillengewinne, Zuordnung zur BRD, zur DDR bzw. zur Mannschaft des wiedervereinigten Deutschlands sowie einige aus diesen Angaben errechenbare Variablen (z.B. Alter bei erster Olympiateilnahme, Anzahl der Olympiateilnahmen) und liegen in SPSS (Version 25, IBM, Armonk, N.Y.) vor. - Tab. 1 beinhaltet die deskriptive Beschreibung der Gesamtzahl der Olympiateilnehmerinnen und -teilnehmer, differenziert nach der Zugehörigkeit zur jeweiligen Olympiamannschaft (OM), die Beschreibung der Verstorbenen und deren Lebensdauer, einschließlich des Anteils der Verstorbenen an den Olympiamannschaften sowie des Verhältnisses zwischen Medaillengewinnern und verstorbenen Medaillengewinnern.

Es wurden für die Teilkohorten Überlebenswahrscheinlichkeiten (ggf. in mehreren Aggregationsstufen) ermittelt und miteinander mittels WilcoxonGehan-Signifikanztest verglichen (Bühl, 2019, 823). Zusätzlich werden Unterschiede zwischen den Teilkohorten mit dem Kaplan-Meier-Verfahren (Gaus \& Muche, 2014, $197 \mathrm{ff}$.) berechnet und auf signifikante Differenzen mittels LogRank-Test geprüft, um die Unterschiedsbefunde mit einem zweiten Standardverfahren abzusichern (zur Differenz 


\begin{tabular}{|c|c|c|c|c|c|c|c|c|c|c|c|c|}
\hline \multirow[b]{3}{*}{$N$} & \multicolumn{3}{|c|}{$\begin{array}{l}\text { Alle Mitglieder OM } 1956 \text { bis } \\
2016\end{array}$} & \multicolumn{3}{|c|}{$\begin{array}{l}\text { Mitglieder OM } 1992 \text { bis } \\
2016^{\mathrm{a}}\end{array}$} & \multicolumn{3}{|c|}{$\begin{array}{l}\text { Mitglieder OM BRD } 1956 \text { bis } \\
1988\end{array}$} & \multicolumn{3}{|c|}{$\begin{array}{l}\text { Mitglieder OM DDR } 1956 \text { bis } \\
1988\end{array}$} \\
\hline & Alle & Weibl & Männl & Alle & Weibl & Männl & Alle & Weibl & Männl & Alle & Weibl & Männl \\
\hline & 6066 & 1959 & 4107 & 2325 & 981 & 1344 & 2107 & 478 & 1629 & 1634 & 500 & 1134 \\
\hline \multicolumn{13}{|c|}{ Verstorbene (V) und deren Lebensdauer (LD) } \\
\hline $\mathrm{N}_{V}$ & 400 & 37 & 363 & 7 & 1 & 6 & 275 & 21 & 254 & 118 & 15 & 103 \\
\hline Anteil $N_{v}$ in \% & 6,59 & 1,89 & 8,84 & 0,30 & 0,10 & 0,45 & 13,05 & 4,39 & 15,59 & 7,22 & 3,00 & 9,08 \\
\hline $\begin{array}{l}\text { Min. LD (Mo- } \\
\text { nate) }\end{array}$ & 234 & 234 & 293 & 234 & 234 & 317 & 282 & 282 & 293 & 341 & 584 & 341 \\
\hline $\begin{array}{l}\text { Max. LD (Mo- } \\
\text { nate) }\end{array}$ & 1118 & 1088 & 1118 & 584 & 234 & 584 & 1104 & 1088 & 1104 & 1118 & 1088 & 1104 \\
\hline $\begin{array}{l}\text { MW LD (Mo- } \\
\text { nate) }\end{array}$ & 788,0 & 740,6 & 792,8 & 405,3 & 234,0 & 433,8 & 803,7 & 770,3 & 806,4 & 774,1 & 757,7 & 780,1 \\
\hline $\begin{array}{l}\text { Sta.AW LD } \\
\text { (Monate) }\end{array}$ & 183,1 & 222,7 & 178,3 & 129,7 & - & 115,6 & 178,6 & 244,3 & 172,5 & 171,0 & 120,1 & 173,6 \\
\hline \multicolumn{13}{|c|}{ Medaillengewinner $\left(V_{M G}\right)$ und verstorbene Medaillengewinner $\left(V_{M G}\right)$} \\
\hline $\mathrm{N}_{M G}$ & 2071 & 715 & 1356 & 685 & 285 & 400 & 611 & 130 & 481 & 775 & 300 & 475 \\
\hline $\mathrm{V}_{\mathrm{MG}}$ & 138 & 18 & 120 & 2 & 0 & 2 & 89 & 9 & 80 & 47 & 9 & 38 \\
\hline $\begin{array}{l}\text { Anteil } V_{M G} \text { in } \\
\%\end{array}$ & 6,66 & 2,52 & 8,85 & 0,29 & 0 & 0,50 & 14,57 & 6,92 & 16,63 & 6,06 & 3,00 & 8,00 \\
\hline
\end{tabular}

zwischen Wilcoxon-Gehan und LogRank Martinez \& Naranjo, 2010). Mit Hilfe von Cox-Regressionen wird anschließend untersucht, welche der im Datensatz enthaltenen Variablen wie stark das relative Sterberisiko (HazardRatio) beeinflussen (Gaus \& Muche, 2014, 384 ff.; Schumacher \& Schmoor, 2002, 90 ff.; Zwiener, Blettner, \& Hommel, 2011). Die verwendeten Verfahren sind Standardprozeduren der medizinischen Statistik (z. B. Altman, 1999, 365 ff.; Bland, 2015, $251 \mathrm{ff}$; Klein \& Moeschberger, 2003, 295 ff.; Ziegler, Lange, \& Bender, 2004).

Die für den Vergleich mit der Gesamtbevölkerung notwendigen Daten stammen aus der Human Mortality Database (HMD), einem Kooperationsprojekt, an dem u.a. das Max-PlanckInstitut für Demografie Rostock und die University of California, Berkeley, maßgeblich beteiligt sind und dessen Daten bis zum Geburts- bzw. Sterbejahr 2017 sich unter www.mortality.org finden ${ }^{5}$. HMD vereint Geburts- und Sterbedaten

\footnotetext{
${ }^{5}$ Daher kann bei Vergleichen mit der Gesamtbevölkerung auch nur der Zeitraum bis einschließlich 2017 betrachtet werden.
}

der Gesamtbevölkerung verschiedener Länder aus offiziellen und historischen Quellen und konstruiert aus diesen u.a. Mortalitätsraten und Sterbetafeln („life tables“; zur Methodik Wilmoth, Andreev, Jdanov, Glei, \& Riffe, 2019), die den Standard für versicherungsmathematische Berechnungen darstellen. Der Vergleich zwischen den Daten für die Gesamtbevölkerung und die der Olympiateilnehmerinnen und -teilnehmer erfolgt anhand der jeweiligen standardisierten Mortalitätsraten („standardised mortality ratio“ [SMR]).

Die Analysestrategie besteht damit im Aggregieren der Mortalitätsraten der deutschen Olympiateilnehmerinnen und -teilnehmer für Vergleiche mit der Gesamtbevölkerung $^{6}$, in der Testung auf Unterschiede der Lebensspannen

\footnotetext{
${ }^{6}$ Am aussagekräftigsten wären Sterbetafeln analog zur den Sterbetafeln der Gesamtbevölkerung, also Sterbewahrscheinlichkeiten für jedes Lebensalter im betreffenden Kalenderjahr. Derartige Sterbetafeln liefern aber in der Gruppe der Olympiateilnehmerinnen und -teilnehmer keine Aussage, da es nur geringe Besetzungen pro Lebensjahr und ggf. gar keine Todesfälle gibt. Daher wurden mehrere Lebensalter und mehrere Kalenderjahre solange aggregiert, bis
}

zwischen interessierenden Gruppen mittels Wilcoxon-Gehan-Signifikanztest der Überlebenswahrscheinlichkeiten sowie anhand des Kaplan-Meier-Schätzer mit Log-Rank-Test. Um im Rahmen des verfügbaren Datensatzes nach Hinweisen auf Ursachen für detektierte Unterschiede zu suchen, werden Cox-Regressionen gerechnet und interpretiert. Entlang dieses Vorgehens wird nach Befunden gesucht, die die aus dem Forschungsstand abgeleiteten Thesen prüfen.

\section{Ergebnisse}

\section{Standardisierte Mortalitätsrate deutscher Olympiateilnehmer}

Von den insgesamt 6066 deutschen Teilnehmerinnen und -teilnehmern an Olympischen Spielen der Jahre 1956 bis 2016 starben bis zum Stichtag 01.07.2019 genau 400. Für den Vergleich mit der Gesamtbevölkerung wurden aus den $\mathrm{Ge}$ burtsjahren der Olympiateilnehmenden

die jeweiligen zeitraum- und altersgruppenspezifischen Risikogruppen eine vertretbare Größe bei genügend hohen Sterbefällen erreicht haben. 
Tab. 2 Altersgruppen- und zeitraumspezifische SMR differenziert nach alter Bundesrepublik und ehemaliger DDR

\begin{tabular}{|c|c|c|c|c|c|c|}
\hline \multirow{2}{*}{$\begin{array}{l}\text { Zeitraum/ } \\
\text { Alters- } \\
\text { gruppe }\end{array}$} & \multicolumn{3}{|c|}{$\begin{array}{l}\text { Olympiamannschaft BRD bezogen } \\
\text { auf Bevölkerung Westdeutschland }\end{array}$} & \multicolumn{3}{|c|}{$\begin{array}{l}\text { Olympiamannschaft DDR bezogen } \\
\text { auf Bevölkerung Ostdeutschland }\end{array}$} \\
\hline & $\begin{array}{l}\text { Beobachtete } \\
\text { Todesfälle }\end{array}$ & $\begin{array}{l}\text { Erwartete } \\
\text { Todesfälle }\end{array}$ & SMR & $\begin{array}{l}\text { Beobachtete } \\
\text { Todesfälle }\end{array}$ & $\begin{array}{l}\text { Erwartete } \\
\text { Todesfälle }\end{array}$ & SMR \\
\hline \multicolumn{7}{|l|}{ 1956-1974 } \\
\hline $15-34$ & 10 & 7,48 & 1,34 & 0 & 5,43 & - \\
\hline $35-64$ & 5 & 8,04 & 0,62 & 2 & 3,49 & 0,57 \\
\hline $65+$ & 2 & 0,71 & 2,80 & \multicolumn{3}{|c|}{ Keine Risikogruppe } \\
\hline \multicolumn{7}{|l|}{ 1975-1994 } \\
\hline $15-34$ & 13 & 5,86 & 2,22 & 9 & 5,77 & 1,56 \\
\hline $35-64$ & 40 & 40,44 & 0,99 & 21 & 34,36 & 0,61 \\
\hline $65+$ & 18 & 12,84 & 1,40 & 2 & 2,49 & 0,80 \\
\hline \multicolumn{7}{|l|}{$1995-2017$} \\
\hline $15-34$ & 4 & 0,37 & 10,71 & 1 & 0,35 & 2,86 \\
\hline $35-64$ & 96 & 47,26 & 2,03 & 63 & 47,61 & 1,32 \\
\hline $65+$ & 271 & 239,57 & 1,13 & 103 & 152,29 & 0,68 \\
\hline \multicolumn{7}{|c|}{$\begin{array}{l}\text { ¿Lesebeispiel: In den Jahren } 1956 \text { bis } 1974 \text { sind in der Altersgruppe der 15- bis 34-Jährigen } 10 \\
\text { Personen gestorben. Bei einer Mortalitätsrate wie in der westdeutschen Gesamtbevölkerung wären } \\
\text { 7,48 Todesfälle zu erwarten gewesen }\end{array}$} \\
\hline
\end{tabular}

die Größe der jeweiligen Risikogruppe („exposure group“) sowie die beobachteten Todesfälle (Sterbetafeln) ab 1956 für jedes Alter gebildet. Die beobachtete Anzahl der Sterbefälle der deutschen Olympiateilnehmerinnen und -teilnehmer wurde mit den aus der Mortalitätsrate der Gesamtbevölkerung und aus der Größe der Gruppe der Olympiateilnehmenden („exposure group“) errechneten erwarteten Todesfälle ins Verhältnis gesetzt und so die Mortalitätsrate (SMR) ermittelt. Da bei gegebener Größe der Risikogruppe und der zu verzeichnenden Todesfälle jahresweise Kombinationen ohne Risikogruppe bzw. Todesfälle häufiger zu beobachten waren, erfolgte eine sukzessive Ausweitung sowohl der Zeitperiode als auch der Altersgruppen. Das Aggregieren zu drei Zeitperioden (1956 bis 1974, 1975 bis 1994 und 1995 bis 2017) und vier Altersgruppen (0-14 Jahre, 15-34 Jahren, 35-64 Jahre, ab 65 Jahren) erbrachte aussagekräftige Werte, wobei kürzere Zeitperioden und Altersgruppen mit geringerer Spannweite bei Bedarf und Verfügbarkeit zusätzliche Evidenz liefern. Die Altersklasse 0-14 Jahre wurde dabei nicht betrachtet, da die Olympiateilnahme einen Tod vor dem Erreichen des 14. Lebensjahres de facto ausschließt und damit kein Vergleich zur jeweiligen Gesamtbevölkerung erfolgen kann.

\section{Westdeutsche Olympia- teilnehmende}

Vergleicht man die Olympiateilnehmerinnen und -teilnehmer, die die Bundesrepublik Deutschland in den gemeinsamen deutschen Olympiamannschaften 1956 bis 1964 und/oder in den selbstständigen Mannschaften 1968 bis 1988 vertraten, mit der Bevölkerung auf dem Gebiet der westdeutschen Bundesländer, so ist in der Altersgruppe der 15- bis 34-Jährigen eine erhöhte und bis zur jüngsten Zeitperiode (1995 bis 2017) steigende standardisierte Mortalitätsrate gegenüber der Gesamtbevölkerung zu erkennen. Statt der erwarteten gut sieben Todesfälle wurden im Zeitraum 1956 bis 1974 zehn Todesfälle $\left(\mathrm{SMR}_{\mathrm{w} 15-34 ; 56-74}=134 \%\right), 1975$ bis 1994 statt knapp sechs erwarteten 13 Todesfälle $\left(\mathrm{SMR}_{\mathrm{w} 15-34 ; 75-94}=222 \%\right)$ und 1995 bis 2017 statt knapp 0,4 vier Todesfälle beobachtet. Rechnerisch führt dies $\mathrm{zu}$ einer $\mathrm{SMR}_{\mathrm{w} 15-34 ; 95-17}$ von $1071 \%$ (vgl. Spalten 2, 3 und 4 in $\bullet$ Tab. $2^{7}$ ).

\footnotetext{
7 In • Tab. 2 sind die SMR aus Platzgründen als Quotient und nicht als Prozentsatz angegeben.
}

Im Altersbereich der 35- bis 64Jährigen liegt das Sterberisiko in den Perioden 1956 bis 1974 sowie 1975 bis 1994 unterhalb der Gesamtbevölkerung $\left(\mathrm{SMR}_{\mathrm{w} 35-64 ; 56-74}=62 \%\right.$; $\mathrm{SMR}_{\mathrm{w} 35-64 ; 75-94=}=$ $99 \%)$, ist für die jüngste Periode jedoch doppelt so hoch $\left(\mathrm{SMR}_{\mathrm{w} 35-64 ; 96-2017}=203 \%\right.$; - Tab. 2, Spalte 4, Zeilen 4, 8 und 12), was ausschließlich auf die Männer zurückzuführen ist. Bei den 65-Jährigen und Älteren sinkt hingegen das Sterberisiko im Zeitverlauf. Das im Zeitraum 1956 bis 1974 noch knapp dreifache Risiko für die westdeutschen Olympiateilneh-

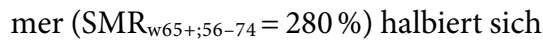
$\left(\mathrm{SMR}_{\mathrm{w} 65+; 75-94}=140 \%\right)$ und übersteigt das Risiko der Gesamtbevölkerung im jüngsten Zeitraum nur noch in geringem Maße $\left(\mathrm{SMR}_{\mathrm{w} 65+; 95-17}=113 \%\right.$; vgl. - Tab. 2, Spalte 4, Zeilen 4, 9 und 13).

\section{Ostdeutsche Olympia- teilnehmende}

Für die ostdeutschen Olympiateilnehmerinnen und -teilnehmer wurden im Vergleich zur Bevölkerung in der DDR bzw. den ostdeutschen Bundesländern für die Altersgruppe der 15- bis 34-Jährigen ebenfalls erhöhte Mortalitätsrisiken ermittelt, die jedoch in beiden Fällen maßgeblich von den Männern induziert wurden, aber mit SMR ${ }_{\mathrm{o} 15-34 ; 75-94}=156 \%$ und $\mathrm{SMR}_{\mathrm{o} 15-34 ; 95-17}=286 \%$ unterhalb der Werte der Olympiateilnehmenden aus den alten Bundesländern lagen. 35- bis 64-Jährige ostdeutsche Olympioniken weisen in den frühen Perioden eine gegenüber der ostdeutschen Gesamtbevölkerung verringerte Mortalitätsrate auf $\left(\mathrm{SMR}_{035-64 ; 56-74}=57 \%\right.$; $\left.\mathrm{SMR}_{035-64 ; 75-94}=61 \%\right)$, die jedoch im Zeitraum von 1995 bis 2017 deutlich über dem Wert in der Gesamtbevölkerung lag $\left(\mathrm{SMR}_{035-64 ; 95-17}=132 \%\right.$; vgl. - Tab. 2, Spalte 7). In der Altersgruppe der 65-Jährigen und Älteren liegen sowohl im Zeitraum 1975 bis 1994 als auch 1995 bis 2017 die Mortalitätsraten unter denen der Gesamtbevölkerung $\quad\left(\mathrm{SMR}_{\mathrm{o} 65+; 75-94}=80 \%\right.$ bzw. $\mathrm{SMR}_{\mathrm{o} 65+; 95-17}=68 \%$; - Tab. 2, Spalte 7, Zeilen 9 und 13).

\section{Deutsche Teilnehmende ab 1992}

Bei den Olympiateilnehmerinnen und -teilnehmern, die ihre ersten Olympi- 


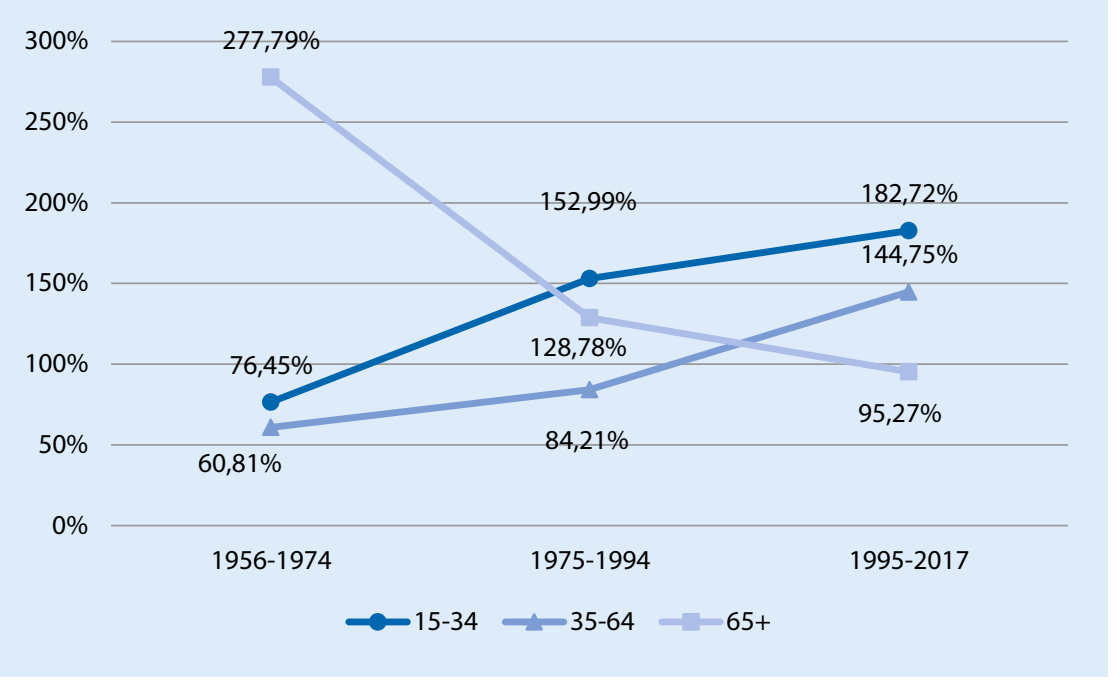

Abb. 1 A Entwicklung der standardisierten Mortalitätsraten (SMR) nach Altersgruppen und Zeitperioden

schen Spiele in einer Mannschaft des wiedervereinten Deutschlands erlebten $(n=2325)$, sind bis einschließlich 30.06.2019 nur 7 Todesfälle zu beklagen. Dennoch ist die SMR im Vergleich mit der Gesamtbevölkerung leicht erhöht $\left(\mathrm{SMR}_{\mathrm{G} 15-34 ; 95-17}=119 \%\right)^{8}$. Für den $\mathrm{Al}-$ tersbereich von 35 bis 64 deutet sich eine geringere Mortalitätsrate als in der Gesamtbevölkerung an. Aufgrund des in manchen Altersgruppen und Zeiträumen geringen Umfangs der Risikogruppen sowie der hohen Bevölkerungszahlen mit entsprechend hoher Zahl an Todesfällen, ergeben sich geringe Konfidenzintervalle für die Sterbewahrscheinlichkeit und in Folge dessen auch für die Zahl der erwarteten Todesfälle sowie für die SMR der Risikogruppe. Zufallsereignisse haben in der Risikogruppe daher auch eine stärkere Bedeutung für die SMR als in der Gesamtbevölkerung. - Tab. 2 enthält die Gesamtübersicht über die alters- und zeitraumspezifischen SMR der

\footnotetext{
8 Bei den sieben zu beklagenden Todesfällen handelt es sich in zwei Fällen um eine Krebserkrankung, in zwei Fällen um plötzlichen Herztod und um einen Verkehrsunfall während der Trainertätigkeit bei Olympischen Spielen. Zudem starbeine Snowborderin bei einer Brandkatastrophe einer Gletscherbahn. In einem Fall wird als Todesursache lediglich "lange schwere Krankheit" genannt. Hätten sich statt der beobachteten sieben Todesfällen nur sechs ereignet, ergäbe sich eine $S_{M R} R_{G 15-34 ; 95-17}=102 \%$.
}

entsteht statt der in 0 Abb. 1 dargestellten $3 \times 3$-Felder-Matrix eine Matrix aus acht Altersgruppen und sechs Zeitintervallen. Die daraus resultierenden Regressionsgeraden steigen für die beiden Altersgruppen der 15- bis 24- sowie der 25- bis 34-Jährigen im Zeitverlauf, sinken aber für die sechs nachfolgenden Altersbereiche.

Dies deutet darauf hin, dass sich für die jüngeren Altersgruppen das Mortalitätsrisiko der Athletinnen und Athleten im Vergleich zur Gesamtbevölkerung intertemporär negativ entwickelt, während es in den höheren Altersgruppen in Richtung Gesamtbevölkerung konvergiert. Allerdings erreichen in der $8 \times 6$-Felder-Matrix aus Altersgruppen und Zeitintervallen von den besetzten 36 Feldern nur sieben SMR-Werte von kleiner 1 bzw. $100 \%$, sodass auch im Zeitverlauf sinkende Regressionsgeraden in den älteren Altersgruppen nur in Richtung einer Normalisierung des Mortalitätsrisikos weisen. Die deutlichen Nachteile beim Sterberisiko für olympische Athletinnen und Athleten bleiben jedoch bestehen.

Alles in allem widerspricht eine Vielzahl von SMR und deren intertemporale Entwicklung der These T1, sodass diese zurückgewiesen werden muss.

\section{Anaerob vs. aerob geprägte Sportarten}

Die verschiedenen olympischen Sportarten beanspruchen die metabolischen Stoffwechselprozesse in unterschiedlichen Relationen (Kenney, Wilmpore, \& Costill, 2015). Daher wird nach Auffälligkeiten zwischen den verschiedenen Sportarten gesucht, die geeignet sind, T2 zu falsifizieren. Als Prototypen anaerob geprägter Sportarten sollen dabei die leichtathletischen Wurfdisziplinen $(n=186 ; 18$ Sterbefälle) und Gewichtheben $(n=79 ; 9$ Sterbefälle) gelten. Als aerob geprägte Sportarten werden Skilanglauf $(n=123 ; 11$ Sterbefälle) und Rudern ( $n=542$; 24 Sterbefälle) klassifiziert.

Die SMR zwischen beiden Gruppen betragen $\mathrm{SMR}_{\mathrm{arob}}=153 \%$ bzw. 
$\mathrm{SMR}_{\text {anaerob }}=79 \%{ }^{9}$. Die Berechnung der Überlebenswahrscheinlichkeit mit Hilfe der Wilcoxon-Gehan-Statistik (Gehan, 1965) erbringt keine signifikanten Unterschiede im direkten Vergleich zwischen den jeweiligen Sportarten sowie im Gesamtvergleich $(p>0,05)^{10}$. Verbindet man Wurf und Gewichtheben zur Klasse „anaerobe Sportarten“ und vergleicht diese mit der Gruppe aus Skilanglauf und Rudern als aerobe Sportarten, so ergibt sich ebenfalls kein signifikanter Unterschied. Zu gleichen Ergebnissen führt das Kaplan-Meier-Verfahren. Mit Hilfe des Log-Rank-Tests lassen sich ebenso keine Unterschiede zwischen den als anaerob bzw. aerob klassifizierten Sportarten sichern $(p>0,05)$. Dies gilt auch für einen Vergleich zwischen den leichtathletischen Wurfdisziplinen und den anderen Disziplinen der Leichtathletik ( $n=759 ; 63$ Sterbefälle). Als Ergebnis einer Cox-Regression wäre demzufolge zu erwarten, dass die Differenzierung zwischen aeroben und anaeroben Sportarten keinen Beitrag zur Erklärung der Gesamtüberlebenszeit der Olympiateilnehmerinnen und -teilnehmer in den leichtathletischen Wurfdisziplinen, dem Gewichtheben, dem Skilanglauf und dem Rudern ( $n=930 ; 62$ Sterbefälle) liefert.

Fügt man daher Geschlecht, Alter bei erstmaliger Olympiateilnahme, die Unterscheidung zwischen Sommer- und Winterspielen, das Jahr der erstma-

9 Bei Bildung der 95\%-Konfidenzintervalle für die erwarteten Todesfälle ergeben sich überschneidende obere und untere Grenzen für die aeroben Sportarten von $144 \%<S_{M R} R_{a e r o b}<54 \%$ sowie von $278 \%<\mathrm{SMR}_{\text {anaerob }}<105 \%$ für die anaeroben Sportarten.

10 Bei den verwendeten Daten handelt es sich um Vollerhebungen bezüglich der an den Start gegangenen Gruppen sowie der in diesen Gruppen aufgetretenen Todesfälle. Dennoch werden die $p$-Werte berichtet, da das Ereignis "Tot eines Individuums aus einem Kollektiv" als eine Stichprobe verstanden wird, die die „Realisierung einer konkreten Wirklichkeit aus einer unendlichen Vielzahl potenziell möglicher Wirklichkeiten" (Behnke, 2005; =-3f.) darstellt. Ausführliche Hinweise zur Entscheidung, unter welchen Umständen bei Vollerhebungen die $p$ Werte berichtet werden sollen finden sich bei Behnke (2005) sowie Broscheid und Gschwend (2005). ligen Olympiateilnahme ${ }^{11}$, die Unterscheidung zwischen DDR, BRD und Gesamtdeutschland, die Differenzierung zwischen Einzelsportarten, MixedSportarten und Mannschaftssportarten, die Anzahl der Olympiateilnahmen sowie die Anzahl der gewonnenen Medaillen ${ }^{12}$ neben der Unterscheidung zwischen den einzelnen als aerob bzw. anaerob klassifizierten Sportarten als mögliche Risikofaktoren in eine Cox-Regression (Einschlussverfahren) ein, so erweist sich lediglich das Geschlecht mit einem Hazard-Ratio von $\mathrm{HR}_{\mathrm{G}}=4,39^{13}(p<0,01)$ sowie die Differenzierung zwischen Einzel- und Mixsportarten ${ }^{14}\left(\mathrm{HR}_{\mathrm{M}}=2,57\right.$; $p<0,05)$ als signifikant risikobeeinflussend, nicht jedoch die Art der ausgeübten Sportarten (• Tab. 3).

Ersetzt man die Differenzierung zwischen den betrachteten vier Sportarten bzw. Disziplinen durch die Unterscheidung zwischen aeroben (Skilanglauf, Rudern) und anaeroben Sportarten (leichtathletischer Wurf, Gewichtheben), so ist der metabolische Status ebenfalls kein signifikanter Risikofaktor.

Da keinerlei Hinweise gefunden werden konnten, die die These T2 stützen und die vorliegenden Befunde T2 widersprechen, muss T2 zurückgewiesen werden.

\section{Einzel- vs. Mannschaftssportarten}

Mannschaftssportarten zeichnen sich gegenüber Einzelsportarten durch eine Interaktion der beteiligten Sportlerinnen und Sportler aus, die wesentlich über eine additive Verknüpfung von

\footnotetext{
11 Mit dem Jahr der ersten Olympiateilnahme und dem Alter bei erstmaliger Olympiateilnahme wird auch das Geburtsjahr determiniert, sodass dieses nicht als Kovariable berücksichtigt wird.

12 DieVerwendungderAnzahl dergewonnenen Gold-, Silber- und Bronzemedaillen als separate Variablen verändern das Ergebnis nicht.

13 Dies bedeutet inhaltlich, dass Athleten der betrachteten Gruppe ein auf $439 \%$ erhöhtes Hazard-Risiko gegenüber Athletinnen aufweisen. Allerdings ist das $95 \%$-Konfidenzintervall mit 1,57 (untere Grenze) bis 10,43 (obere Grenze) recht breit.

${ }^{14}$ Vgl. zur Differenzierung zwischen Mannschafts-, Mix- und Einzelsportarten Abschn.4.3.
}

Einzelleistungen, die typischer Weise bei Staffeln oder bei Mannschaftswertungen vorliegen, hinausgehen. Entsprechend dieses Kriteriums wurden als olympische Mannschafssportarten Basketball, Beach-Volleyball, Bobsport, Curling, Fußball, Handball, Hockey, Eishockey, Segeln, Volleyball und Wasserball klassifiziert ( $n=3099 ; 222$ Sterbefälle). Die Sportarten Badminton, Eiskunstlauf, Kanurennsport, Radsport, Rennrodeln, rhythmische Sportgymnastik, Rudern, Synchronschwimmen, Tennis, Tischtennis, Trampolin und Wasserspringen ( $n=1445 ; 77$ Sterbefälle) vereinen sowohl Einzel- als auch Mannschaftssportwettbewerbe. Dagegen sind Alpiner Skisport, Biathlon, Bogenschießen, Boxen, Eisschnelllauf, Fechten, Freestyle, Gewichtheben, Golf, Judo, Leichtathletik, moderner Fünfkampf, nordische Kombination, Reiten, Ringen, Schwimmen, Short-Track, Skeleton, Skilanglauf, Skispringen, Snowboarding, Sportschießen, Taekwondo, Triathlon und Turnen ( $n=1522 ; 101$ Sterbefall) im Sinne dieser Analyse Einzelsportarten. Bezogen auf die Gesamtkohorte ergeben sich standardisierte Mortalitätsraten von 109\% in den Mannschafts-, $81 \%$ in den Mixund $100 \%$ in den Einzelsportarten. ${ }^{15}$

Weder zusammengefasst über alle drei Sportartenkategorien, noch im paarweisen Vergleich finden sich signifikante Unterschiede in den jeweiligen Überlebensfunktionen oder nach dem Kaplan-Meier-Verfahren $(p>0,05)$. Auch eine CoxRegression $^{16}$ mit den oben genannten möglichen Risikofaktoren erbringt keine Hinweise auf ein erhöhtes Risikopotenzial einer der gebildeten Sportartenkategorien (• Tab.4). T3 muss daher zurückgewiesen werden.

15 Bei Beachtung des $95 \%$-Konfidenzintervalls für die erwarteten Todesfälle ergeben sich Spannen von $96 \%<\mathrm{SMR}_{\text {Mannschaft }}<125 \%$, $68 \%<$ SMR $_{\text {Mix }}<100 \%$ bzw. $84 \%<$ SMREinzel $<$ $124 \%$.

16 Die Cox-Regression in diesem Abschnitt unterscheidet sich von der im Abschn. 4.2 vorgenommenen Cox-Regression durch deren Bezugaufalle 6066 Beobachtungsfälle. 
Tab. 3 Cox-Regression ( $n=930$ bei 62 Sterbefällen) zur Bestimmung des Risikoeinflusses aerober bzw. anaerober Sportarten (Regressionskoeffizient B, zugehöriger Standardfehler SE, Prüfgröße Wald, Freiheitsgrad df, $p$-Wert des zugehörigen Tests, untere und obere Grenze des 95 \% Konfidenzintervalls für das relative Risiko, wenn die entsprechende Variable um eine Einheit erhöht wird)

\begin{tabular}{|c|c|c|c|c|c|c|c|c|}
\hline & \multirow[t]{2}{*}{ B } & \multirow[t]{2}{*}{ SE } & \multirow[t]{2}{*}{ Wald } & \multirow[t]{2}{*}{ Df } & \multirow[t]{2}{*}{$\begin{array}{l}\text { Signifi- } \\
\text { kanz }\end{array}$} & \multirow[t]{2}{*}{$\operatorname{Exp}(B)$} & \multicolumn{2}{|c|}{$\begin{array}{l}95 \% \text { Konfidenzintervall für } \\
\operatorname{Exp(B)}\end{array}$} \\
\hline & & & & & & & Untere & Obere \\
\hline Geschlecht & 1,479 & 0,489 & 9,169 & 1 & 0,002 & 4,390 & 1,685 & 11,436 \\
\hline Alter bei erstmaliger Olympiateilnahme & $-0,007$ & 0,042 & 0,026 & 1 & 0,873 & 0,993 & 0,915 & 1,079 \\
\hline Olympische Sommer- oder Winterspiele & 0,242 & 0,388 & 0,391 & 1 & 0,532 & 1,274 & 0,596 & 2,725 \\
\hline Anzahl an Teilnahmen & $-0,034$ & 0,171 & 0,039 & 1 & 0,843 & 0,967 & 0,691 & 1,352 \\
\hline Anzahl an Medaillen & 0,419 & 0,220 & 3,628 & 1 & 0,057 & 1,520 & 0,988 & 2,338 \\
\hline Einzel-, Mix- oder Mannschaftssportarten & 0,945 & 0,442 & 4,578 & 1 & 0,032 & 2,573 & 1,083 & 6,116 \\
\hline Deutschland nach Wiedervereinigung & - & - & 3,943 & 2 & 0,139 & - & - & - \\
\hline BRD & 1,028 & 1,163 & 0,782 & 1 & 0,376 & 2,797 & 0,286 & 27,317 \\
\hline DDR & 0,505 & 1,157 & 0,191 & 1 & 0,662 & 1,657 & 0,172 & 15,985 \\
\hline Jahr der erstmaligen Olympiateilnahme & $-0,020$ & 0,019 & 1,083 & 1 & 0,298 & 0,981 & 0,945 & 1,017 \\
\hline $\begin{array}{l}\text { Aerob (Skilanglauf, Rudern) vs. anaerobe } \\
\text { (Wurf, Gewichtheben) Sportarten }\end{array}$ & $-0,139$ & 0,420 & 0,110 & 1 & 0,740 & 0,870 & 0,382 & 1,980 \\
\hline
\end{tabular}

\begin{tabular}{|c|c|c|c|c|c|c|c|c|}
\hline & \multirow[t]{2}{*}{ B } & \multirow[t]{2}{*}{ SE } & \multirow[t]{2}{*}{ Wald } & \multirow[t]{2}{*}{ Df } & \multirow[t]{2}{*}{$\begin{array}{l}\text { Signifi- } \\
\text { kanz }\end{array}$} & \multirow[t]{2}{*}{$\operatorname{Exp}(B)$} & \multicolumn{2}{|c|}{$\begin{array}{l}95 \% \text { Konfidenzintervall für } \\
\text { Exp(B) }\end{array}$} \\
\hline & & & & & & & Untere & Obere \\
\hline Geschlecht & 0,781 & 0,178 & 19,302 & 1 & 0,000 & 2,183 & 1,541 & 3,093 \\
\hline Alter bei erstmaliger Olympiateilnahme & 0,017 & 0,009 & 3,226 & 1 & 0,072 & 1,017 & 0,998 & 1,036 \\
\hline Olympische Sommer- oder Winterspiele & $-0,160$ & 0,126 & 1,615 & 1 & 0,204 & 0,852 & 0,666 & 1,090 \\
\hline Anzahl an Teilnahmen & $-0,047$ & 0,081 & 0,332 & 1 & 0,564 & 0,954 & 0,815 & 1,118 \\
\hline Anzahl an Medaillen & 0,199 & 0,060 & 10,999 & 1 & 0,001 & 1,220 & 1,085 & 1,372 \\
\hline Mannschaftssportarten & - & - & 0,705 & 2 & 0,703 & - & - & - \\
\hline Einzelsportarten & 0,095 & 0,125 & 0,576 & 1 & 0,448 & 1,099 & 0,861 & 1,404 \\
\hline Mixsportarten & 0,020 & 0,158 & 0,016 & 1 & 0,899 & 1,020 & 0,749 & 1,390 \\
\hline Deutschland nach Wiedervereinigung & - & - & 16,055 & 2 & 0,000 & - & - & - \\
\hline BRD & 0,495 & 0,447 & 1,227 & 1 & 0,268 & 1,641 & 0,683 & 3,943 \\
\hline DDR & 0,052 & 0,449 & 0,014 & 1 & 0,907 & 1,054 & 0,437 & 2,540 \\
\hline Jahr der erstmaligen Olympiateilnahme & $-0,028$ & 0,007 & 14,785 & 1 & 0,000 & 0,973 & 0,959 & 0,987 \\
\hline
\end{tabular}

\section{Ostdeutsche vs. westdeutsche Olympiateilnehmende}

Beim Vergleich der Überlebensfunktionen zwischen den Olympiateilnehmerinnen und -teilnehmern aus der ehemaligen DDR $(n=1634 ; 118$ Sterbefälle) und der alten Bundesrepublik ( $n=2107 ; 275$ Sterbefälle), die erstmalig bei den olympischen Spielen 1956 bis 1988 teilnahmen, lassen sich mit der Wilcoxon-Gehan-Statistik signifikante Unterschiede $(p<0,01)$ sichern. Die Olympiateilnehmer der DDR haben eine höhere Überlebenserwartung. Zum selben Ergebnis gelangt man bei Anwendung des Log-Rank-Tests innerhalb der Kaplan-Meier-Methode $\quad(p<0,01$; - Abb. 2).

Sucht man mittels Cox-Regression nach Risikofaktoren für die Überlebenswahrscheinlichkeit und bezieht alle im Abschn. „Methoden“ eingeführten Variablen ein, sollte sich die Zuordnung der erstmaligen Olympiateilnahme zur ehemaligen DDR, zur alten Bundesrepublik bzw. zum wiedervereinigten Deutschland ( $n=2325 ; 7$ Sterbefälle) als Prädikator erweisen. Es zeigt sich, dass die Zuordnung zur alten Bundesrepublik ein signifikanter Risikofaktor $(p<0,01)$ ist. Im Vergleich zur erstmaligen Teilnahme für die DDR ist das HazardRatio auf $164 \%$ und im Vergleich zur erstmaligen Teilnahme für das wiedervereinte Deutschland auf 105\% erhöht. Allerdings liegen die 95\%-Konfidenzintervalle zwischen $68 \%$ und $394 \%$ im Vergleich mit der DDR bzw. zwischen $43 \%$ und $254 \%$ im Vergleich zum wiedervereinten Deutschland. Dennoch stellt die Beteiligung für die $\mathrm{BRD}$ nach dem Geschlecht $\left(\mathrm{HR}_{\mathrm{G}}=2,18 ; p<0,01\right)$ den zweistärksten Risikofaktor im Modell dar. Weitere signifikante Risikofaktoren sind die Gesamtzahl der Medaillen $\left(\mathrm{HR}_{\mathrm{M}}=1,22 ; p<0,01\right)$ sowie das Jahr der ersten Olympiateilnahme $\left(\mathrm{HR}_{\mathrm{OT}}=0,97\right.$; $p<0,01)$ (vgl. ๑ Tab. 4).

Bezieht man in das Modell nur die Olympiateilnehmerinnen und -teilneh- 


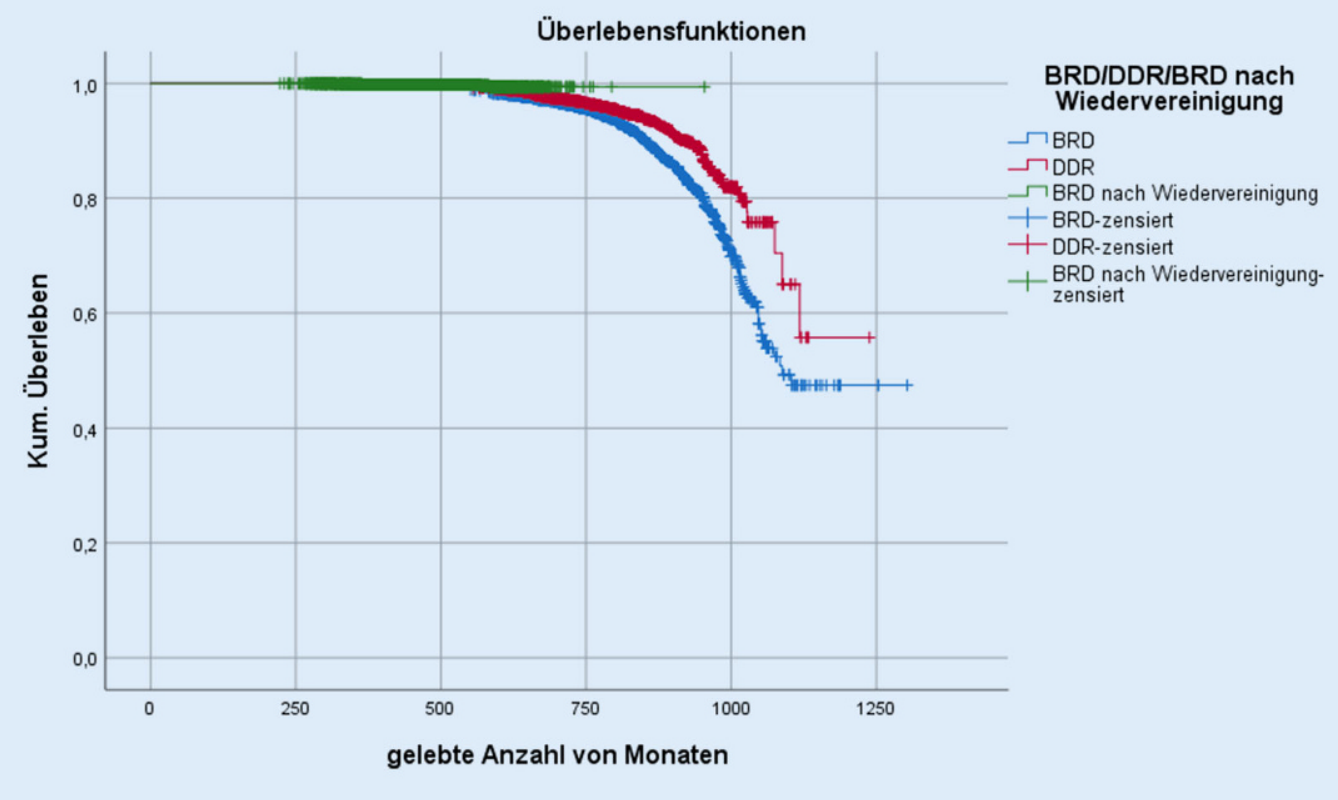

Abb. $2 \triangleleft$ Kaplan-MeierKurve für die deutschen Olympiateilnehmer

mer der ehemaligen DDR und der alten Bundesrepublik ein, so ergibt sich für die bundesdeutschen Olympioniken ein um $55 \%$ erhöhtes Hazard-Ratio. Bedingt durch die Extraktion von 2325 Athletinnen und Athleten mit erstmaliger Olympiateilnahme für das wiedervereinte Deutschland und deren bislang geringer Anzahl an Sterbefällen verringert sich das $95 \%$-Konfidenzintervall deutlich auf $124 \%$ bis $192 \%$. Das Sterberisiko ist demnach für die westdeutschen Athletinnen und Athleten im Vergleich zu den Ostdeutschen signifikant erhöht.

Führt man die Cox-Regression separat für die ehemalige DDR sowie für die alte Bundesrepublik durch, sind beide Modelle zwar vergleichbar, verfügen aber über leicht unterschiedliche Ausprägungen der signifikanten Risiken. Einflussreichster signifikanter Risikofaktor ist in beiden Fällen das Geschlecht $\quad\left(\mathrm{HR}_{\mathrm{G} \_\mathrm{BRD}}=2,28 ; \quad p<0,01\right.$; $\left.\mathrm{HR}_{\mathrm{G}_{-} \mathrm{DDR}}=1,93 ; \quad p<0,05\right)$, gefolgt von der Anzahl gewonnener Medaillen $\left(\mathrm{HR}_{\mathrm{M} \_\mathrm{BRD}}=1,19 ; p<0,05 ; \mathrm{HR}_{\mathrm{M} \_\mathrm{DDR}}=1,26\right.$; $p<0,05)$. Zudem verringert für die alte Bundesrepublik eine spätere erstmalige Olympiateilnahme das Risiko signifikant $\left(\mathrm{HR}_{\mathrm{T} \_\mathrm{BRD}}=0,97 ; p<0,01\right)$, während dies für die DDR keine signifikante Veränderung des Risikos bedeutet.

Auch die Bildung der SMR aus beiden deutschen Risikogruppen, den danach erwarteten und den tatsächlichen Todesfällen deutet auf einen Überlebensvorteil der DDR-Olympioniken hin. Deren SMR liegt differenziert nach drei Zeiträumen und drei Alterskohorten stets unterhalb der SMR der westdeutschen Olympiateilnehmerinnen und -teilnehmer (• Tab. 5, Spalte 5). In fünf der acht zu betrachtenden Fälle ${ }^{17}$ liegt die obere Grenze des 95\%-Konfidenzintervalls der SMR für die ostdeutschen Olympiateilnehmerinnen und -teilnehmer unterhalb der unteren Grenze des 95\%-Konfidenzintervalls der westdeutschen Teilnehmerinnen und Teilnehmer (•Tab. 5, Spalte 7). Die Hypothese H4 ist daher nicht nur zurückzuweisen, für weitergehende Untersuchungen sollte zudem von einem höheren Mortalitätsrisiko der westdeutschen Olympiateilnehmerinnen und -teilnehmer ausgegangen werden.

\section{Sportartspezifische Mortalität infolge vermuteter Dopinganwendung}

Um die Sportarten mit vermuteter Dopinganwendung in Bezug auf ihr spezifisches Sterberisiko zu beurteilen, wurden Schwimmen ( $n=411 ; 14$ Sterbefälle), Biathlon ( $n=76 ; 4$ Sterbefälle), Skilanglauf

17 In einem Fall existiert nur eine westdeutsche Risikogruppe, sodass kein Vergleich möglich ist.
( $n=124 ; 11$ Sterbefälle), Leichtathletik ( $n=943 ; 79$ Sterbefälle), Eiskunstlauf ( $n=119 ; 4$ Sterbefälle), Rudern ( $n=542 ; 24$ Sterbefälle) und Gewichtheben $(n=79 \text {; } 9 \text { Sterbefälle })^{18}$ mit der Summe aller anderen Sportarten $(n=3772$; 255 Sterbefälle) verglichen. Die SMR der einzelnen mutmaßlich dopingbelasteten Sportarten verglichen mit den bislang weniger auffälligen Sportarten enthält - Tab. 6.

Hinsichtlich der Überlebenswahrscheinlichkeit ergeben sich lediglich signifikante Unterschiede zwischen Gewichtheben und Schwimmen $(p<0,01)$ sowie zwischen Gewichtheben und den anderen Sportarten $(p<0,05)$. Es finden sich keine Unterschiede, wenn man die Sportarten mit vermuteter Dopinganwendung in Summe den weniger in Verdacht stehenden Sportarten gegenüberstellt. Die Wilcoxon-GehanStatistik im Kaplan-Meier-Verfahren detektiert lediglich den Unterschied zwischen Schwimmen und Gewichtheben $(p<0,05)$.

Fügt man dem bislang verwendeten Cox-Regressionsmodell die differenzierten dopingbelasteten Sportarten als

18 Dies sind die Sportarten, auf deren Dopingbelastung in der Begründung zum ersten Dopingopfer-Hilfegesetz explizit Bezug genommen wird (Bundestag, 2002b, 5). 


\begin{tabular}{|c|c|c|c|c|c|c|}
\hline $\begin{array}{l}\text { Betrachtungszeitraum/ } \\
\text { Geburtenjahrgänge }\end{array}$ & $\begin{array}{l}\text { Mannschafts- } \\
\text { zugehörigkeit }^{\mathrm{a}}\end{array}$ & $\begin{array}{l}\text { Bis } 30.06 .2019 \text { beobachtete } \\
\text { Todesfälle }^{b}\end{array}$ & $\begin{array}{l}\text { Erwartete To- } \\
\text { desfälle }\end{array}$ & SMR & $\begin{array}{l}\text { SMR untere } \\
\text { Grenze }^{c}\end{array}$ & $\begin{array}{l}\text { SMR obere } \\
\text { Grenze }\end{array}$ \\
\hline \multicolumn{7}{|l|}{$1956-1974$} \\
\hline \multirow[t]{2}{*}{ 15-34 (1922-1959) } & BRD & 244 & 195,32 & 1,25 & 1,10 & 1,44 \\
\hline & DDR & 112 & 160,68 & 0,70 & 0,60 & 0,80 \\
\hline \multirow[t]{2}{*}{ 35-64 (1892-1939) } & BRD & 165 & 142,85 & 1,16 & 1,01 & 1,33 \\
\hline & DDR & 59 & 81,15 & 0,73 & 0,64 & 0,84 \\
\hline 65 und älter (bis 1909) & BRD/DDR & Keine DDR-Risikogruppe & & & & \\
\hline \multicolumn{7}{|l|}{$1975-1994$} \\
\hline \multirow[t]{2}{*}{ 15-34 (1941-1979) } & BRD & 100 & 86,78 & 1,15 & 0,96 & 1,45 \\
\hline & DDR & 59 & 72,22 & 0,82 & 0,68 & 1,03 \\
\hline \multirow[t]{2}{*}{ 35-64 (1911-1959) } & BRD & 259 & 204,84 & 1,26 & 1,12 & 1,45 \\
\hline & DDR & 112 & 166,16 & 0,68 & 0,60 & 0,77 \\
\hline \multirow[t]{2}{*}{65 und älter (bis 1929) } & BRD & 67 & 64,67 & 1,04 & 0,91 & 1,21 \\
\hline & DDR & 14 & 16,33 & 0,86 & 0,75 & 1,00 \\
\hline \multicolumn{7}{|l|}{$1995-2017$} \\
\hline \multirow[t]{2}{*}{$15-34(1961-2002)^{d}$} & BRD & 12 & 10,03 & 1,20 & 0,65 & 2,19 \\
\hline & DDR & 5 & 6,97 & 0,72 & 0,39 & 1,32 \\
\hline \multirow[t]{2}{*}{ 35-64 (1931-1992) } & BRD & 196 & 164,67 & 1,19 & 1,04 & 1,39 \\
\hline & DDR & 101 & 132,33 & 0,76 & 0,67 & 0,89 \\
\hline \multirow[t]{2}{*}{65 und älter (bis 1952) } & BRD & 238 & 194,37 & 1,22 & 1,09 & 1,41 \\
\hline & DDR & 90 & 133,63 & 0,67 & 0,60 & 0,77 \\
\hline \multicolumn{7}{|c|}{$\begin{array}{l}\text { "Die Mannschaftszugehörigkeit „BRD“ umfasst die Olympioniken, die zum Zeitpunkt ihrer ersten Olympiateilnahme die Staatsbürgerschaft der BRD } \\
\text { innehatten und für die gemeinsame deutsche Mannschaft (1956-1964) oder für die Mannschaft der Bundesrepublik (1968-1988) antraten. Entsprechendes } \\
\text { gilt für die Mannschaftszugehörigkeit „DDR“ } \\
\text { 'Lesebeispiel: Die Geburtenjahrgänge } 1922 \text { bis } 1959 \text { waren in den Jahren } 1956 \text { bis } 1974 \text { zwischen } 15 \text { und } 34 \text { Jahre alt. Bis zum } 30.06 .2019 \text { verstarben von } \\
\text { den westdeutschen Olympiateilnehmern dieser Jahrgänge 243. Bezogen auf die Risikogruppe der Olympiateilnehmer aus der BRD und der DDR wären } \\
\text { 194,82 Todesfälle zu erwarten gewesen } \\
\text { 'Basierend auf dem 95\%-Konfidenzintervall der erwarteten Todesfälle } \\
\text { dAuf Grund der geringen Zahl der Todesfälle wurde hier das empirische Konfidenzintervall berechnet (Bosch, 2000, 53 ff.) }\end{array}$} \\
\hline
\end{tabular}

\begin{tabular}{|c|c|c|c|c|c|c|c|}
\hline & Schwimmen & Biathlon & Skilanglauf & Leichtathletik & Eiskunstlauf & Rudern & Gewichtheben \\
\hline Erwartete Todesfälle & 26,43 & 5,12 & 8,47 & 66,80 & 7,92 & 35,05 & 5,42 \\
\hline Beobachtete Todesfälle & 14 & 4 & 11 & 79 & 4 & 24 & 9 \\
\hline SMR & 0,53 & 0,78 & 1,30 & 1,18 & 0,51 & 0,68 & 1,66 \\
\hline SMR untere Grenze & 0,47 & 0,70 & 1,16 & 1,06 & 0,45 & 0,61 & 1,49 \\
\hline SMR obere Grenze & 0,60 & 0,88 & 1,47 & 1,34 & 0,57 & 0,78 & 1,88 \\
\hline
\end{tabular}

weiteren Risikofaktor hinzu, ist mit keiner einzelnen Sportart ein signifikanter Risikoauf- oder -abschlag verbunden. Zum gleichen Ergebnis gelangt man, wenn man die dopingbelasteten Sportarten als Gruppe in die Cox-Regression aufnimmt (• Tab. 7).

Der Hinweis aus einer ggf. unterschiedlichen Überlebensfunktion für die Sportart Gewichtheben reicht jedoch nicht aus, um die These T5, die sich ja auf eine Gruppe von Sportarten mit einem vermuteten Dopingge- brauch bezog, beizubehalten. Wenn ein Dopinggebrauch $\mathrm{zu}$ einer geringeren Überlebensrate führen sollte, würde das Ergebnis nicht für einen unterschiedlichen Dopinggebrauch in den bislang im Fokus stehenden Sportarten gegenüber anderen Sportarten sprechen.

\section{Medaillenspezifische Mortalität}

Um eine medaillenspezifische Mortalität zu identifizieren, wurden zunächst alle Medaillengewinnerinnen und -ge- winner danach kategorisiert, welche Medaillen sie errungen hatten ${ }^{19}$. Zudem wurde die höchste Medaillenkategorie festgehalten. Zusätzlich zum Vergleich zwischen den Medaillengewinnerinnen und -gewinnern wurden auch die Athletinnen und Athleten ohne Medaillenge-

19 Die Gruppen bestanden demnach aus den Gewinnern von Goldmedaillen, von Goldund Silbermedaillen, von Gold-, Silber- und Bronzemedaillen usw. bis hin zur Gruppe derer, die keine Medaille gewannen. 
Tab. 7 Cox-Regression ( $n=6066$ bei 400 Sterbefällen) zur Bestimmung des Risikoeinflusses von Sportarten mit vermuteter Dopingbelastung

\begin{tabular}{|c|c|c|c|c|c|c|c|c|}
\hline & \multirow[t]{2}{*}{ B } & \multirow[t]{2}{*}{ SE } & \multirow[t]{2}{*}{ Wald } & \multirow[t]{2}{*}{ Df } & \multirow[t]{2}{*}{$\begin{array}{l}\text { Signifi- } \\
\text { kanz }\end{array}$} & \multirow[t]{2}{*}{$\operatorname{Exp}(B)$} & \multicolumn{2}{|c|}{$\begin{array}{l}95 \% \text { Konfidenzintervall für } \\
\text { Exp(B) }\end{array}$} \\
\hline & & & & & & & Untere & Obere \\
\hline Geschlecht & 0,784 & 0,178 & 19,424 & 1 & 0,000 & 2,189 & 1,545 & 3,102 \\
\hline Alter bei erstmaliger Olympiateilnahme & 0,017 & 0,009 & 3,427 & 1 & 0,064 & 1,018 & 0,999 & 1,037 \\
\hline Olympische Sommer- oder Winterspiele & $-0,161$ & 0,126 & 1,644 & 1 & 0,200 & 0,851 & 0,665 & 1,089 \\
\hline Anzahl an Teilnahmen & $-0,045$ & 0,081 & 0,316 & 1 & 0,574 & 0,956 & 0,816 & 1,120 \\
\hline Anzahl an Medaillen & 0,201 & 0,060 & 11,210 & 1 & 0,001 & 1,223 & 1,087 & 1,376 \\
\hline Jahr der erstmaligen Olympiateilnahme & $-0,028$ & 0,007 & 14,617 & 1 & 0,000 & 0,973 & 0,959 & 0,987 \\
\hline Vermutete Dopingbelastung & 0,067 & 0,119 & 0,317 & 1 & 0,573 & 1,069 & 0,847 & 1,351 \\
\hline Deutschland nach Wiedervereinigung & - & - & 16,195 & 2 & 0,000 & - & - & - \\
\hline BRD & 0,498 & 0,447 & 1,238 & 1 & 0,266 & 1,645 & 0,685 & 3,953 \\
\hline DDR & 0,052 & 0,449 & 0,014 & 1 & 0,907 & 1,054 & 0,437 & 2,540 \\
\hline Mannschaftssportarten & - & - & 0,480 & 2 & 0,787 & - & - & - \\
\hline Einzelsportarten & 0,068 & 0,134 & 0,261 & 1 & 0,610 & 1,071 & 0,824 & 1,391 \\
\hline Mixsportarten & $-0,010$ & 0,167 & 0,003 & 1 & 0,953 & 0,990 & 0,714 & 1,374 \\
\hline
\end{tabular}

winn herangezogen. Beim Vergleich der Überlebenswahrscheinlichkeiten sind zunächst keine Unterschiede zwischen den verschiedenen Gruppen der Medaillengewinnenden sowie zwischen der Gruppe der Medaillengewinnenden und der Gruppe ohne Medaillengewinn (Wilcoxon-Gehan-Statistik) zu sichern. Differenziert man jedoch lediglich zwischen den höchsten errungenen Medaillenkategorien, dann ergibt sich zwischen den Goldmedaillengewinnenden $(n=816,53$ Sterbefälle) und der Gruppe ohne Medaillenerfolg ( $n=3995$, 262 Sterbefälle) ein signifikanter Unterschied $(p<0,05)$ auf Basis der geschätzten Mittelwerte der Lebensmonate zulasten der Goldmedaillengewinnerinnen und -gewinner, obwohl die SMR beider Gruppen nur geringfügig differieren $\left(\mathrm{SMR}_{\mathrm{Gold}}=0,99 ; \quad \mathrm{SMR}_{\text {nicht }}=1,00\right)$. Der Unterschied erklärt sich vorrangig aus der geringeren Überlebenswahrscheinlichkeit $(p<0,01)$ der Goldmedaillengewinnerinnen und -gewinner der DDR ( $n=331,213$ Sterbefälle) gegenüber der gleichen Gruppe der alten Bundesrepublik ( $n=206,135$ Sterbefälle) sowie der höheren Überlebenswahrscheinlichkeit $(p<0,05)$ der Gruppe ohne Medaillenerfolg aus der DDR ( $n=859,71$ Sterbefälle) gegenüber der der BRD $(n=1496,186$ Sterbefälle). Stellt man nur die Medaillengewinnerinnen und -gewinner den Teilnehmerinnen und Teilnehmern ohne Medaille gegenüber, differieren die
Überlebenswahrscheinlichkeiten signifikant $(p<0,05)$. Das Kaplan-MeierVerfahren bestätigt auch die Ergebnisse der Überlebensanalyse, dass Goldmedaillengewinnerinnen und -gewinner eine geringere Lebenszeit haben als die Gruppe ohne Medaillengewinn. Dieser Befund findet sich auch in der Cox-Regression (Einschlussverfahren) wieder. Die Anzahl der gewonnenen Medaillen ist darin der zweithöchste Risikofaktor $\left(\mathrm{HR}_{\mathrm{M}}=1,22 ; p<0,01\right)$ im Modell (- Tab. 4 und 7, jeweils Zeile „Anzahl an Medaillen"). Werden statt der Zahl der gewonnenen Medaillen die Gruppen der jeweiligen Medaillengewinne, also der Gewinn mindestens einer Gold-, Silberoder Bronzemedaille, ins Modell aufgenommen, so verringert sich das Risiko mit jedem geringer wertigen Medaillenerfolg bis hin zur Medaillenlosigkeit signifikant auf jeweils $93 \%(p<0,01)$. Wird die jeweilige Anzahl der gewonnenen Gold-, Silber- und Bronzemedaillen als separate Variablen im Modell berücksichtigt, führt die Zahl der gewonnenen Silber- $\left(\mathrm{HR}_{\mathrm{M} \_s}=1,26 ; p<0,05\right)$ und Bronzemedaillen ( $\left.\mathrm{HR}_{\mathrm{M} \_\mathrm{B}}=1,35 ; p<0,05\right) \mathrm{zu}$ einer signifikanten Risikosteigerung, nicht jedoch die Zahl der Goldmedaillen $(p>0,05)$. Dies ist ebenso ein Indikator für ein steigendes Risiko bei Zunahme des sportlichen Erfolgs wie der Befund, der sich bei Berücksichtigung der jeweils höchsten errungenen Medaillenkategorie bei Nichtberück- sichtigung der Medaillenanzahl ergibt. Das Risiko sinkt hier mit jeder nicht erreichten Medaillenkategorie auf jeweils $83 \%(p<0,01)$.

Die vorgelegten Befunde stehen der These T6 deutlich entgegen. Stützende Befunde konnten nicht erbracht werden. T6 ist daher zu verwerfen.

\section{Geschlechtsspezifische Mortalität}

Der Vergleich zwischen deutschen Olympiateilnehmerinnen $(n=1959,37$ Todesfälle) und -teilnehmern $(n=4107$, 363 Todesfälle) fördert eine signifikant höhere Überlebenswahrscheinlichkeit $(p<0,01)$ der Olympiateilnehmerinnen $\quad\left(\mathrm{SMR}_{\mathrm{OT} \_ \text {Männer }}=134 \%\right.$; SMR $\left._{\text {OT_Frauen }_{2}}=29 \%\right)$ zu Tage. Dies gilt auch beim separaten Vergleich der Geschlechter in den Mannschaften der BRD $(p<0,01)$ und der DDR $(p<0,05)$. Aufgrund der bislang geringen Anzahl an Todesfällen in den Mannschaften des wiedervereinten Deutschlands kann für die Athletinnen und Athleten, die 1992 und später an ihren ersten Olympischen Spielen teilgenommen haben, derzeit noch keine Aussage getroffen werden. Beim Vergleich der Geschlechter unter Beachtung der ost- bzw. westdeutschen Mannschaftszugehörigkeit fällt auf, dass die Überlebenswahrscheinlichkeit der Frauen nicht $(p>0,05)$, die der Männer aber sehr wohl zu Ungunsten der westdeutschen Männer differiert $(p<0,01)$. 
Beide Ergebnisse werden bei Anwendung des Kaplan-Meier-Verfahrens bestätigt. $\mathrm{Da}$ auch in den bereits berichteten Cox-Regressionen das Geschlecht mit $\mathrm{HR}_{\mathrm{G}}=2,18(p<0,01)$ stets die signifikante erklärende Variable mit dem höchsten Einfluss auf das Risiko war (• Tab. 4 und 7, jeweils Spalte "Geschlecht“) und separate Cox-Regressionen für die BRD $\left(\mathrm{HR}_{\mathrm{G}_{-} \mathrm{BRD}}=2,28 ; p<0,01\right)$ und die DDR $\left(\mathrm{HR}_{\mathrm{G}_{-} \mathrm{DDR}}=1,93 ; \quad p<0,05\right)$ zu keinem anderen Ergebnis kommen, kann mit dem vorliegenden Datensatz die These H7 nicht zurückgewiesen werden: Deutsche Olympiateilnehmerinnen haben im Vergleich zu deutschen Olympiateilnehmern eine geringere Mortalität.

Zur Prüfung von T8 wurde das Verhältnis der kumulierten Sterbewahrscheinlichkeiten der Olympiateilnehmerinnen und -teilnehmer in den Altersgruppen 15 bis 34 Jahre, 35 bis 64 Jahre sowie 65 Jahre und älter jeweils in den Zeiträumen 1956 bis 1974, 1975 bis 1994 sowie 1995 bis 2017 gebildet und mit demselben Verhältnis der Sterbewahrscheinlichkeiten zwischen den Geschlechtern in der Gesamtbevölkerung mittels $\mathrm{T}$-Test verglichen. Infolge fehlender Risikogruppen oder Todesfällen bei den Olympiateilnehmerinnen lassen sich nicht alle Altersgruppen in allen Zeiträumen geschlechtsspezifisch verfolgen, sodass lediglich für die Zeiträume 1975 bis 1994 sowie 1995 bis 2017 jeweils die drei zu betrachtenden altersspezifischen Verhältniswerte vorlagen. Angesichts der daraus resultierenden geringen Fallzahl von $n=6$ ermittelt der T-Test einen $p$-Wert von $p=0,08$. Zusammen mit der Effektstärke von Cohens $\mathrm{d}=1,23$ kann nicht mehr plausibel angenommen werden, dass sich die Verhältniszahlen der Sterbewahrscheinlichkeiten zwischen Frauen und Männern unverändert im Verhältnis der Sterbewahrscheinlichkeiten der Olympiateilnehmerinnen und -teilnehmer niederschlagen. Dies deutet darauf hin, dass das Verhältnis des Mortalitätsrisikos zwischen Frauen und Männern in der Gesamtbevölkerung nicht dem zwischen Olympiateilnehmerinnen und -teilnehmern entspricht. Das zuvor festgestellte erhöhte Mortalitätsrisiko im Vergleich zur Gesamtbevölkerung schlägt sich somit nicht proportional zur Risikoverteilung zwischen den Geschlechtern in der Gesamtbevölkerung nieder.

\section{Diskussion}

Leistungssportlichem Engagement wird eine Reihe gesellschaftspolitisch wertvoller Implikationen nachgesagt und daher auf Ebene von Bund (Spitzensport), Ländern (Nachwuchsleistungssport) und Kommunen (Unterhaltung der Sportstättenbasis) direkt oder indirekt gefördert (Bundestag, 2019; zur Bedeutung nationaler Medaillenerfolge vgl. z. B. Emrich, Gassmann, Haut, Pierdzioch, \& Prohl, 2015). Mit dieser Förderung sollen nicht nur spitzensportliche Erfolge (zur Differenzierung zwischen Erfolg und Leistung vgl. z.B. Emrich \& Pierdzioch, 2018) ermöglicht, sondern auch Nachteile kompensiert werden, die sich beispielsweise aus verzögerten Bildungsabschlüssen oder der Zeitkonkurrenz zwischen einer Erwerbstätigkeit und der Sportausübung ergeben und die nicht über Marktmechanismen, z. B. in Form höherer Einkommen, kompensiert werden. Die vorliegenden Befunde können als Indiz gewertet werden, dass leistungssportliche Karrieren auch den Einsatz von Lebenszeit nach sich ziehen und dieser Effekt mit zunehmendem sportlichen Erfolg steigt. Sie unterstützen damit empirisch, was deutsche Leistungssportlerinnen und -sportler bereits wissen oder zumindest ahnen ${ }^{20}$. Das konkrete Ausmaß der Auswirkungen auf die Lebenszeit der Athletinnen und Athleten und damit letztlich auch die infolge der Vergesellschaftung individueller sportlicher Erfolge zu leistende Kompensation lässt sich auf der Grundlage von Mortalitätsdaten nur mit langer zeitlicher Verzögerung verlässlich feststellen. Im Sinne vorbeugender Risikominimierung unterstreichen die Befunde jedoch die Notwendigkeit einer engen, von Sportverbandsinteressen unabhängigen medizinischen Überwa-

\footnotetext{
${ }^{20}$ Kugelstoß-Weltmeisterin Christina Schwanitz: „Jeder der Leistungssport betreibt weiß, dass er seinen Körper kaputt macht." (Deutschlandfunk, 2018).
}

chung für die derzeitige Generation der Leistungssportlerinnen und -sportler ${ }^{21}$. Zudem sind Programme zur Förderung des Leistungssports ohne ausreichende Berücksichtigung vorsorgender gesundheitlicher Komponenten ethisch nicht (mehr) zu verantworten. In der aktuellen Version des Potenzialanalysesystems PotAS, welches die Grundlagen für die Verteilung der Spitzensportfördermittel des Bundes an die Sportfachverbände liefern soll, findet sich im Bereich „Struktur“ mit dem Attribut „Gesundheitsmanagement“ (Unterattribute „Medizinisches Kompetenzzentrum“, „Ärztliche Betreuung und Physiotherapie“, „Psychologie und Ernährung“, „AntiDoping“ sowie „Prävention von Belästigung und Missbrauch im Sport") neben 12 anderen auch ein entsprechender Indikator, dessen relative Gewichtung im Gesamtgefüge knapp $7 \%$ ausmacht (PotAS, 2019).

Mortalitätsraten einer Risikogruppe geben immer die Summe der gegenüber einer Vergleichsgruppe wirkenden positiven und negativen Faktoren zu einem bestimmten Messzeitpunkt wieder. Die Einnahme von Dopingmitteln als gesundheitlicher Risikofaktor schlägt sich in den sportartspezifischen Vergleichen sowie im Vergleich zwischen den Olympioniken aus der ehemaligen DDR bzw. der alten Bundesrepublik derzeit nicht im erwarteten Maße bzw. der erwarteten Richtung nieder. Insbesondere die signifikant höheren Mortalitätsraten der westdeutschen Olympiateilnehmer sind kontrafaktisch. Mögliche Erklärung für diese Befunde sind (I) eine Neutralisierung des Risikos durch andere, in dieser Untersuchung unbeobachtete Risikofaktoren, (II) vergleichbare sportartübergreifende Dopingpraktiken in Ost und West, (III) komplexe Zusammenhänge leistungssportlicher Karrieren mit sozialen und sozioökonomischen Komponenten, (IV) bislang unbeobachtete Selektionsprozesse durch die unterschiedlichen gesellschaftlichen Bedingungen und die differierenden Leis-

\footnotetext{
21 Diese Argumentation basiert auf der Annahme, dass medizinische Interventionen in Mitglieder der Risikogruppe in Summe zu einer höheren Überlebenswahrscheinlichkeitführen.
} 
tungssportsysteme in beiden deutschen Staaten sowie (V) eine noch nicht hinreichende Anzahl von Sterbefällen. Weitere Erklärungsansätze, wie beispielsweise die unterschiedliche Erfolgswahrscheinlichkeit bei einer Olympiateilnahme sowie ungleiche mediale und gesellschaftliche Verhandlungen von Erfolgen und Misserfolgen, sind möglich. Die vorgelegten Befunde sprechen jedoch dafür, bei der Bewertung gesundheitlicher Wirkungen von Leistungssport nicht nur auf Teilgruppen oder die Praxis des Dopingmissbrauchs abzustellen, sondern alle Leistungssportlerinnen und Leistungssportler als Risikogruppe zu betrachten ${ }^{22}$. Die Wirkungen von Leistungssport auf nationalem und internationalem Niveau auf die Überlebenswahrscheinlichkeit sollten nicht nur unter Hinzuziehung weiterer potenzieller Risikofaktoren wie beispielsweise Todesursachen, Suizid, Karrieredauer oder sozioökonomischer Status untersucht werden, die Ergebnisse sind auch Nachwuchsathletinnen und -athleten zu erläutern, um deren individuelle Entscheidung für oder gegen eine leistungssportliche Laufbahn auf eine informierte Grundlage zu stellen.

Wie alle bisherigen Studien zur Mortalität von Spitzensportlern basiert die Diskussion der vorliegenden Daten auf der Annahme, dass Leistungssport einen Einfluss auf die Lebenserwartung der Risikogruppe hat. Allerdings ist auch denkbar, dass durch den Leistungssport eine Gruppe von Menschen selektiert wird, die infolge ihrer genetischen Dispositionen einen risikoreicheren Lebensstil ausprägen und die so als Gruppe eine geringere Lebenserwartung als die Gesamtbevölkerung aufweist. Leistungssport würde danach eine von verschiedenen Varianten von Lebensstilen darstellen, in

22 Die Deutsche Fußball-Liga, der Deutsche Fußball-Bund und die gesetzliche Unfallversicherung VBG lassen ab 2020 den Gesundheitszustand von mindestens 300 ehemaligen Profi-Fußballerinnen und -fußballern im Alter zwischen 40 und 69 kontrollieren. Die Fußballerinnen und Fußballer werden Probanden in einer nationalen Kohorte von rund 160.000 Personen einer seit 2014 laufenden bevölkerungsbezogenen Langzeitstudie zur Erforschung von Krankheitsursachen („NakoGesundheitsstudie“, Ärztezeitung, 2019). der sich Menschen mit risikoreicheren Dispositionen durchsetzen. Zur Klärung dieses Endogenitätsproblems wären wohl Zwillingsstudien notwendig, bei dem ein Zwilling Leistungssport betreibt. Als sicher kann jedoch (vorläufig) gelten, dass der deutsche Leistungssport (Hoch-)Risiko-Karrieren auf Gruppenebene produziert und/oder für eine Gruppe mit risikoreichem Lebensstil attraktiv ist. Diese ist bereit, eine kürzere Lebenszeit für sportlichen Erfolg in Kauf zu nehmen (zum Goldman-Dilemma vgl. z.B. González, Johnson, Fedoruk, Posner, \& Bowers, 2018; zur Glühbirnentheorie vgl. Emrich \& Follert, 2019, S. 251).

\section{Schlussfolgerung, Limitationen und Ausblick}

Im Gegensatz zu anderen Ländern liegt für Deutschland bislang nur eine Studie zur Mortalität von Leistungssportlern, nämlich für die deutschen Fußball-Nationalspieler der Jahre 1908 bis 2006 $(n=812)$, vor (Kuss et al., 2011). Gerade die temporäre Existenz zweier deutscher Staaten mit unterschiedlichen (Leistungs-)Sportsystemen sowie die aktuelle Diskussion um die DDRDopingopfer auch 30 Jahre nach dem Mauerfall lässt Mortalitätsdaten von Leistungssportlern sowohl zur Erkenntnisgewinnung als auch zur Anreicherung aktueller Debatten geeignet erscheinen. Genutzt wurde ein eigens zusammengestellter Datensatz mit allen deutschen Olympiateilnehmern und -teilnehmerinnen der Jahre 1956 bis $2016(n=6066)$. Bis einschließlich 30.06.2019 waren davon 400 Olympiateilnehmerinnen und -teilnehmer verstorben. Aus dem rezipierten Forschungsstand wurden sieben Thesen gewonnen, die sich auf die Mortalität im Vergleich zur Gesamtbevölkerung (H1), die Risikofaktoren metabolischer Stoffwechsel (H2), Sportartencharakteristik (H3), Doping (H4, H5), olympischer Erfolg (H6) und Geschlecht (H7, H8) bezogen.

Die Annahmen einer höheren Überlebensrate verglichen mit der Gesamtbevölkerung ( $\mathrm{H} 1)$, einer Risikodifferenz zwischen anaeroben und aeroben Sportarten (H2), zwischen Mannschafts-, Mixbzw. Einzelsportarten (H3) sowie einem höheren Risiko beim Gewinn der Silbermedaille infolge Verlust des Sieges (H6) bestätigten sich nicht. Weiter zeigt sich entgegen der Annahmen, dass der olympische Erfolg ein lineares Risiko für die Überlebenswahrscheinlichkeit darstellt (H6). Zudem lassen sich Differenzen infolge eines vermuteten stärkeren Dopinggebrauchs in bestimmten Sportarten (H4) sowie in der DDR (H5) nicht nachweisen. Dagegen stellt das Geschlecht annahmegemäß einen signifikanten Risikofaktor dar (H7), der nicht der Risikoverteilung zwischen Frauen und Männern in der Gesamtbevölkerung folgt (H8).

Als stärkster Risikofaktor für die Überlebenswahrscheinlichkeit erwies sich das männliche Geschlecht, gefolgt von der Zuordnung zur Mannschaft der alten Bundesrepublik (1956 bis 1964 innerhalb der gemeinsamen deutschen Mannschaft) sowie vom olympischen Erfolg. Die Ergebnisse wurden hinsichtlich ihrer Implikationen auf die Gestaltung aktueller Leistungssportfördersysteme sowie in Bezug auf den Umgang mit leistungssportlichen Risiken diskutiert. Festzuhalten ist jedoch, dass für die beschriebenen Befunde derzeit kein theoretischer Rahmen zur deren Erklärung verfügbar ist, sodass die vorgenommenen Interpretationen ex post vorgenommen werden mussten.

Wie jede Überlebensanalyse sind die vorgelegten Befunde infolge der Verwendung zensierter Daten limitiert. Zudem kann nicht vollständig ausgeschlossen werden, dass Todesfälle übersehen wurden. Allerdings ist es wenig plausibel anzunehmen, dass es zu einer systematischen Verzerrung in Richtung einer der verwendeten Vergleichskategorien (z.B. Herkunft, Geschlecht, Sportart) gekommen ist. Nicht erkannte Todesfälle würden daher zuerst $\mathrm{zu}$ einem noch höheren Risiko für Leistungssportlerinnen und -sportlern gegenüber der Gesamtbevölkerung führen. Auf Grund der verfügbaren Daten konnten nur einige sportspezifische Risikofaktoren kontrolliert werden. Vernachlässigt werden mussten dagegen die in allgemeinen Überlebensanalysen einflussstarken sozioökonomischen, status- und bildungsspezifischen Risikofaktoren, weil 
diese für die Masse der deutschen Olympiateilnehmer ebenso wie Suizid nicht verfügbar waren. Darüber hinaus stellt das oben diskutierte, ungelöste Endogenitätsproblem ebenfalls eine Limitation der vorgelegten Ergebnisse dar.

Die teilweise kontrafaktischen Befunde verlangen einerseits nach einer Wiederholung der Untersuchung mit dann aktualisierten Daten und laden andererseits dazu ein, die betrachtete Gruppe der Olympiateilnehmerinnen und -teilnehmer sowohl zu spezifizieren als auch auszuweiten, um die Reichweite der vorgelegten Befunde zu erweitern. Vor dem Hintergrund staatlicher Leistungssportförderung ist es zudem aus Respekt vor den Leistungssportlerinnen und Leistungssportlern als Risikogruppe angezeigt, die Entwicklung von Modellen zu fördern, die eine Prognose zur Entwicklung der die Sterbewahrscheinlichkeit beeinflussenden Risikofaktoren im Leistungssport gestatten. Wenn individuelle sportliche Leistungen weiterhin gesellschaftlich angeeignet werden, sind deren Erbringer für die entgangene Lebenszeit zu entschädigen.

\section{Korrespondenzadresse}

Prof. Dr. Lutz Thieme
Hochschule Koblenz
Joseph-Rovan-Alle 2,
53424 Remagen, Deutschland
thieme@rheinahrcampus.de

Funding. Open Access funding provided by Projekt DEAL.

\section{Einhaltung ethischer Richtlinien}

Interessenkonflikt. L. Thieme gibt an, dass kein Interessenkonflikt besteht.

Für diesen Beitrag wurden von den Autoren keine Studien an Menschen oder Tieren durchgeführt. Für die aufgeführten Studien gelten die jeweils dort angegebenen ethischen Richtlinien.

Open Access. Dieser Artikel wird unter der Creative Commons Namensnennung 4.0 International Lizenz veröffentlicht, welche die Nutzung, Vervielfältigung, Bearbeitung, Verbreitung und Wiedergabe in jeglichem Medium und Format erlaubt, sofern Sie den/die ursprünglichen Autor(en) und die Quelle ordnungsgemäß nennen, einen Link zur Creative Commons Lizenz beifügen und angeben, ob Änderungen vorgenommen wurden.
Die in diesem Artikel enthaltenen Bilder und sonstiges Drittmaterial unterliegen ebenfalls der genannten Creative Commons Lizenz, sofern sich aus der Abbildungslegende nichts anderes ergibt. Sofern das betreffende Material nicht unter der genannten Creative Commons Lizenz steht und die betreffende Handlung nicht nach gesetzlichen Vorschriften erlaubt ist, ist für die oben aufgeführten Weiterverwendungen des Materials die Einwilligung des jeweiligen Rechteinhabers einzuholen.

Weitere Details zur Lizenz entnehmen Sie bitte der Lizenzinformation auf http://creativecommons.org/ licenses/by/4.0/deed.de.

\section{Literatur}

Abel, E., \& Kruger, M.L. (2005). Longevity or major league baseball players. Research in Sport Medicine, 13(1), 1-5.

Abel, E., \& Kruger, M. L. (2006). The healthy worker effect in major league baseball revisited. Research in Sport Medicine, 14(1), 83-87.

Altman, D.G. (1999). Practical statistics for medical research. Boca Raton:Chapman \& Hall.

Antero-Jacquemin, J., Desgorces, F. D., Dor, F., Sedeaud, A., Haïda, A., LeVan, P., \& Toussaint, J.-F. (2014). Row for your life: a century of mortality follow-up of French Olympic rowers. PLoS One. https://doi. org/10.1371/journal.pone.0113362.

Antero-Jacquemin, J., Rey, G., Marc, A., Dor, F., Haïda, A., Marck, A., Berthelot, G., Calmat, A., Latouche, A., \& Toussaint, J.-F. (2015). Mortality in female and male French Olympians. American Journal of Sports Medicine, 43(6), 1505-1512. https://doi. org/10.1177/0363546515574691.

Ärztezeitung (2019). Fußballprofis nehmen an Nako-Studie teil. https://www.aerztezeitung. de/Politik/Fussballprofis-nehmen-an-NakoStudie-teil-404419.html. Zugegriffen: 27. Nov. 2019.

Beaglehole, R., \& Stewart, A. (1983). The longevity of international rugby players. The New Zealand Medical Journal, 96(735), 513-515.

Becker, D. J., Chay, K. Y., \& Swaminathan, S. (2007). Mortality and the baseball hall of fame: an investigation into the role of status in life expectancy. iHEA 2007 6th world congress: explorations in health economics paper. https:// papers.ssrn.com/sol3/papers.cfm?abstract_ id=995034. Zugegriffen: 4 . Juni 2019.

Behnke, J. (2005). Lassen sich Signifikanztests auf Vollerhebungenanwenden?Einigeessayistische Anmerkungen. Politische Vierteljahresschrift, 46(1), 0-1-0-15.

Belli, S., \& Vanacore, N. (2005). Proportionate mortality of Italien soccer players: is amyotrophic lateral sclerosis an occupational disease? European Journal of Epidemiology, 20(3), 237-242.

Blair, S. N., Kohl III, H., Barlow, C. E., Paffenbarger Jr, R. S., Gibbons, L. W., \& Macera, C. A. (1995). Changes in physical fitness and all-cause mortality. A prospective study of healthy and unhealthy men.Journal of the American Medical Association Network Open, 273(14), 1093-1098. https://doi. org/10.1001/jama.1995.03520380029031.

Bland, M. (2015). An introduction to medical statistics. Oxfort: Oxfort University Press.

Boltanski, I. (1976). Die soziale Verwendung des Körpers. In D. Kamper \& V. Rittner (Hrsg.), Zur Geschichte des Körpers (S. 138-183). München: Hanser.
Bosch, K. (2000). Elementare Einführung in die angewandte Statistik. Braunschweig: Vieweg.

Broscheid, A., \& Gschwend, T. (2005). Zur statistischen Analyse von Vollerhebungen. Politische Vierteljahresschrift, 46(1), 0-16-0-26.

Bühl, A. (2019). SPSS. Einführung in die moderne Datenanalyse ab SPSS 25. Hallbergmoos: Pearson.

Bundestag (2002b). Entwurf eines Gesetzes über eine finanzielle Hilfe für Dopingopfer der DDR (Dopingopfer-Hilfegesetz-DOHG). Drucksache 14/9022. http://dip21.bundestag.de/dip21/ btd/14/090/1409022.pdf. Zugegriffen: 7. Juli 2019.

Bundestag (2016). Zweites Gesetz über eine finanzielle Hilfe für Dopingopfer der DDR (Zweites Dopingopfer-Hilfegesetz). https://beck-online.beck. de/?vpath=bibdata\%2Fges\%2FDOHG\%5F2 \%2Fcont\%2FDOHG\%5F2\%2Ehtm. Zugegriffen: 3. Juli 2019.

Bundestag (2019). 14. Sportbericht der Bundesregierung.Drucksache 19/9150.dip21.bundestag.de/ dip21/btd/19/091/1909150.pdf. Zugegriffen: 8 . Aug. 2019.

Bundestag (2002a). Gesetz über die finanzielle Hilfe für Doping-Opfer der DDR (Dopingopfer-Hilfegesetz - DOHG). Bundesgesetzblatt, Jahrgang 2002 Teill, Nr.62. (S. 3410-3411)

Clarke, P., Walter, S., Hayen, A., Mallon, W., Heijmans, J., \& Studdert, D. (2012). Survival of the fittest: retrospective cohort study of the longevity of Olympic medallists in the modern era. British Medicine Journal, 345, e8308-e8308.

Coate, D., \& Sun, R. (2012). Survival estimates for elite male and female Olympic athletes and tennis championship competitors. Scandinavian Journal of Medicine \& Science in Sports, 23(6), 722-727.

DeKosky, S., Jaffee, M., \& Bauer, R. (2018). Long-term mortality in NFL professional football players. No significant increase, but questions remain. Journal of the American Medical Association, 319(8), 773-775.

Deutschlandfunk (2018). „Ich glaube, viele Politiker haben nie Sport gemacht". Christina Schwanitz und Clemens Prokop im Gespräch mit Matthias Friebe. https://www. deutschlandfunk.de/kugelstosserin-schwanitzvor-der-em-ich-glaube-viele.892.de.html? dram:article_id=424537. Zugegriffen: 8 . Aug 2019.

Eagleton, J.R., McKelvie, S. J., \& de Man, A. (2007). Extraversion and neuroticism in team sport participants, individual sport participants, and nonparticipants. Perceptual and Motor Skills, 105(1), 100-111.

Emrich, E., \&Follert, F. (2019). Eigenplagiate aus ökonomischer Sicht. Eine institutionenökonomische Betrachtung. ORDO, 70(1), 239-255.

Emrich, E., \& Pierdzioch, C. (2018). Modellierung und Bedeutung nationaler Medaillenerfolge bei Olympischen Spielen. In A. Güllich \& M. Krüger (Hrsg.), Sport in Kultur und Gesellschaft. Berlin, Heidelberg: Springer. https://doi.org/10.1007/ 978-3-662-53385-7_10-1.

Emrich, E., Gassmann, F., Haut, J., Pierdzioch, C., \& Prohl, R. (2015). Medaillen für die nationale Repräsentanz? Zur Bedeutung von Medaillenerfolgen bei Olympischen Spielen. Sport und Gesellschaft 12(1), 39-67.

Gajewski, A., \& Poznańska, A. (2008). Mortality of top athletes, actors and clergy in Poland: 1924-2000 follow-up study of the long term effect of physical activity. European Journal of 
Epidemiology, 23(5), 335-340. https://doi.org/ 10.1007/s10654-008-9237-3.

Garatachea, N., Santos-Lozano, A., Sanchis-Gomar, F., Fiuza-Luces, C., Pareja-Galeano, H., Emanuele, E., \& Lucia, A. (2014). Elite athletes live longer than the generalpopulation: a meta-analysis. Mayo Clinic Proceedings, 89(9), 1195-1200.

Gaus, W., \& Muche, R. (2014). Medizinische Statistik. Stuttgart: Schattauer.

Gehan, E. A. (1965). A generalized Wilcoxon test for comparing arbitrarily singly-censored samples. Biometrika, 52(1/2), 203-223.

González, J. M., Johnson, F. R., Fedoruk, M., Posner, J., \& Bowers, L. (2018). Trading health risks for glory: a reformulation of the Goldman dilemma. Sports Medicine, 48(8), 1963-1969. https://doi.org/10. 1007/s40279-018-0881-9.

Kalwij, A. (2018). The effects of competition outcomes on health: evidence from the lifespans of U.S. Olympic medalists. Economics and Human Biology, 31(C), 276-286. https://doi.org/10. 1016/j.ehb.2018.10.001.

Keller, K. (2019). Life expectancy of olympic wrestling champions in comparison to the general population. Journal of Community Health, 44(1), 61-67.

Kenney, W.L., Wilmore, J.H., \& Costill, D. L. (2015). Physiology of sport and exercise (6. Aufl.). Champaigen: Human Kinetics.

Klein, J. P. (2003). Survival analysis. New York: Springer.

Knuth, J. (2018). Dopingopfer gegen Dopingopfer. https://www.sueddeutsche.de/sport/dopinghilfe-ddr-1.4242814. Zugegriffen: 3. Juli 2018.

Kujala, U. M., Kaprio, J., Sarna, S., \& Koskenvuo, M. (1998). Relationship of leisure-time physical activity and mortality. Journal of the American Medical Association, 279(6), 440-444.

Kujawska, A., Topka, W., Gajos, M., Androsiuk-Perkowska, J., Perkowski, R., Husejko, J., Marszałek, A., Szczęśniak, Ż., Cieślińska, A., Skierkowska, N., Przybysz, B., Kwolik, D., Siekacz, D., Rybarczyk, D. \& Kujawski, S. (2017). Do former elite athletes live longer? Potential role of critical window(s) in the development of the health-oriented behaviors and physiological adaptations. Journal of Education, Health and Sport, 7(9), 11-21. https://doi. org/10.5281/zenodo.853509.

Kuss, O., Kluttig, A., \& Greiser, K. H. (2011). Longevity of Soccer Players - An Investigation of all German Internationals from 1908-2006. Scandinavian Journal of Medicine \& Science in Sports, 21(6), e260-e265. https://doi.org/10.1111/j.16000838.2010.01269.x

Last, J., \& Weisser, B. (2015). Der Einfluss von moderater sportlicher Aktivität und Alter auf Kraft, Ausdauer und Gleichgewicht im Erwachsenenalter. Deutsche Zeitschrift für Sportmedizin, 66(1), 5-11.

Lee-Heidenreich, J., Lee-Heidenreich, D., \& Myers, J. (2017). Differences in life expectancy between olympic high jumpers, discus throwers, marathon and 100 meter runners. BMC Sport Science, Medicine and Rehabilitation. https://doi.org/10. 1186/s13102-017-0067-z.

Lehman, E. J., Hein, M. J., Baron, S. L., \& Gersic, C. M. (2012). Neurodegenerative causes of death among retired National Football League players. Neurology, 79(19), 1970-1974.

Leive, A. (2018). Dying to win? Olympic gold medals and longevity. SSRN working paper. https:// ssrn.com/abstract=2410519. Zugegriffen: 8 . Juli 2019.

Lemez, S., \&Baker, J. (2015). Doeliteathleteslivelonger? A systematic review of mortality and longevity in elite athletes. Sport Medicine-Open. https:// doi.org/10.1186/s40798-015-0024-x.

Lemez, S., Wattie, N., \& Baker, J. (2018). The end game: mortality outcomes in North American professional athletes. Scandinavian Journal of Medicine \& Science in Sports, 28(6), 1722-1730.

Lin, Y., Gajewski, A., \& Poznańska, A. (2016). Examining mortality risk and rate of ageing among Polish Olympic athletes: a survival follow-up from 1924 to 2012. British Medicine Journal Open. https:// doi.org/10.1136/bmjopen-2015-010965.

Lindqvist, A.-S., Moberg, T., Ehrnborg, C., Eriksson, B. O., Fahlke, C., \& Rosén, T. (2014). Increased mortality rate and suicide in Swedish former elite male athletes in power sports. Scandinavian Journal of Medicine \& Science in Sports, 24(6), 1000-1005.

Mackay, D. F., Russell, E. R., Stewart, K., MacLean, J. A Pell, J. P., \& Stewart, W. (2019). Neurodegenerative disease mortality among former professional soccer players. New England Journal of Medicine, 381(19), 1801-1808. https://doi.org/10.1056/ NEJMoa1908483.

Marijon, E., Tafflet, M., Antero-Jacquemin, J., El Helou, N., Berthelot, G., Celermajer, D. S., Bougouin, W. Combes, N., Hermine, O., Rey, J.-P., Empana, G., Toussaint, J.-F., \& Jouven, X. (2013). Mortality of French participants in the Tour de France (1947-2012). European Heart Journal, 34(40), 3145-3150. https://doi.org/10.1093/eurheartj/ eht347.

Markowitz, J.S. (2019). Mortality among hispanic and African-American players after desegregtion in major league baseball. Cham: Springer.

Martinez, R. L. M. C., \& Naranjo, J. D. (2010). A pretest for choosing between logrank and wilcoxon tests in two-sample problem. Metron. International Journal of Statistics, 68(2), 111-125.

Medvec, V., Madey, S., \& Gilovich, T. (1995). When less is more: counterfactual thinking and satisfaction among olympic medalists. Journal of Personality and Social Psychology, 69(4), 603-610.

Nguyen, V. T.,Zafonte, R. D., Chen, J. T., Kponee-Shovein, K. Z., Paganoni, S., Pascual-Leone, A., Speizer, F.E., Baggish Jr, A. L., Taylor, H. A., Nadler, L. M., Courtney, T. K., Connor, A., \& Weisskopf, M.G. (2019). Mortality among professional Americanstyle football players and professional American baseball players. Journal of the American Medical Association NetworkOpen,2(5), e194223.https:// doi.org/10.1001/jamanetworkopen.2019.4223.

Pärssinen, M., Kujala, U., Vartiainen, E., Sarna, S. \& Seppäla, T. (2000). Increased premature mortality of competitive powerlifters suspected to have used anabolic agents. International Journal of Sports Medicine, 21(3), 225-227.

PotAS (2019). Attributsystem Sommersportarten. Stand 13.05.2019. https://www.potas.de/dam/ jcr:cdb0c195-40d0-4fec-815e-9c92cdaf28c3/ PotAS_Attributesystem_Sommer2019_20.pdf. Zugegriffen: 8. Aug. 2019.

Radonić, V., Kozmar, D., Počanić, D., Jerkić, H., Bohaček, I., \& Letilović, T. (2017). Mortality and causes of death among Croatian male Olympic medalists. Croatian Medical Journal, 58(4), 263-269.

Ruiz, J., Fiuza-Luces, C., Garatachea, N., \& Lucia, A. L. (2013). Reduced mortality in former elite endurance athletes. International Journal of Sports Physiology and Performance, 9(6), 1046-1049. https://doi.org/10.1123/ijspp.2013-0492.

Schumacher, M., \& Schmoor, C. (2002). Analysen von Ereigniszeiten - Teil II. In M. Schumacher \& G. Schulgen (Hrsg.), Methoden klinischer Studien (S. 89-105). Berlin:Springer.
Sturmberg, J. (2018). Kritik am Dopingopferhilfegesetz.https://www.deutschlandfunk.de/dopingkritik-am-dopingopferhilfegesetz.1346.de. html?dram:article_id=433537. Zugegriffen: 3. Juli 2019.

Sturmberg, J., \& Freitag, D. (2019). „Aufgabe des Staates, dass junge Menschen soziale Sicherheit haben." Dagmar Freitag im Gespräch mit Jessica Sturmberg. https://www.deutschlandfunk. de/sportlerrente-aufgabe-des-staates-dassjunge-menschen.1346.de.html?dram:article id=463639,.Zugegriffen: 15 . Nov. 2019 .

Teramoto, M., \& Bungum, T. J. (2010). Mortality and longevity of elite athletes. Journal of Science and Medicine in Sport, 13(4), 410-416.

Turi-Lynch, B.C., Monteiro, H. L., Fernandes, R. A., Sui, X., Lemes, I.R., \& Codogno, J.S. (2019). Impact of sports participation on mortality rates among Brazilian adults. Journal of Sports Sciences, 37(13), 1443-1448. https://doi.org/10. 1080/02640414.2019.1565109.

Vaupel, J. W. (2010). Biodemography of human ageing Nature, 464, 536-542. https://doi.org/10.1038/ nature 08984

Venkataramani, A., Gandhavadi, M., \& Jena, A. B. (2018). Association between playing American football in the national football league and long term mortality. Journal of the American Medical Association, 319(8), 800-806.

Williams, T. (2012). Long-term mortality effects of an NFL career. Cambridge: MIT. https://economics. mit.edu/files/8461.Zugegriffen: 5. Juli 2019.

Wilmoth, J.R., Andreev, K., Jdanov, D., Glei, D. A., \& Riffe, T. (2019). Methods protocol fort he human mortality database. http://www.mortality.org/ Public/Docs/MethodsProtocol.pdf. Zugegriffen: 25. Nov. 2019

Ziegler, A., Lange, S., \& Bender, R. (2004). Überlebenszeitanalysen:Die Cox-Regression. Deutsche Medizinische Wochenschrift, 129(S03), 1-3.

Zwiener, I., Blettner, M., \& Hommel, G. (2011). Überlebenszeitanalyse. Deutsches Ärzteblatt, 108(10), 163-169. 